\title{
THE FABER POLYNOMIALS FOR ANNULAR SECTORS
}

\author{
JOHN P. COLEMAN AND NICK J. MYERS
}

\begin{abstract}
A conformal mapping of the exterior of the unit circle to the exterior of a region of the complex plane determines the Faber polynomials for that region. These polynomials are of interest in providing near-optimal polynomial approximations in a variety of contexts, including the construction of semiiterative methods for linear equations. The relevant conformal map for an annular sector $\{z: R \leq|z| \leq 1, \theta \leq|\arg z| \leq \pi\}$, with $0<\theta \leq \pi$, is derived here and a recurrence relation is established for the coefficients of its Laurent expansion about the point at infinity. The recursive evaluation of scaled Faber polynomials is formulated in such a way that an algebraic manipulation package may be used to generate explicit expressions for their coefficients, in terms of two parameters which are determined by the interior angle of the annular sector and the ratio of its radii. Properties of the coefficients of the scaled Faber polynomials are established, and those for polynomials of degree $\leq 15$ are tabulated in a Supplement at the end of this issue. A simple closed form is obtained for the coefficients of the Faber series for $1 / z$. Known results for an interval, a circular arc, and a circular sector are reproduced as special cases.
\end{abstract}

\section{INTRODUCTION}

The Faber polynomials for particular regions of the complex plane have been used to provide polynomial and rational approximations in a wide variety of different contexts. Near-minimax polynomial approximations may be obtained by truncating Faber series [15, 9, 1], by economization of Faber series [12] and, for solutions of linear differential equations, by the Lanczos $\tau$-method [2, 3] and by Clenshaw's method [14]. Rational approximations based on Faber series were discussed in [11] and [13], and applications to iterative methods in numerical linear algebra appear in $[8,7,29]$.

The Faber polynomials for any closed bounded continuum $D$ in the complex plane are associated with a certain exterior conformal map. According to the Riemann mapping theorem (e.g., [21, p. 380]) there is a unique function $\phi$, such that

$$
\phi(\infty)=\infty, \quad \lim _{z \rightarrow \infty} \frac{\phi(z)}{z}=1
$$

Received by the editor June 17, 1993.

1991 Mathematics Subject Classification. Primary 30C10, 30C20, 30E10; Secondary 65E05, $65 \mathrm{~F} 10$.

Key words and phrases. Faber polynomials, conformal mapping, annular sector, Faber series, transfinite diameter. 
which maps the complement of $D$ in the extended $z$-plane conformally onto $\{w:|w|>\rho\}$, the complement of a closed disc of radius $\rho$. The number $\rho$ is called the transfinite diameter, or logarithmic capacity, of $D$. The function $\phi$ has a Laurent expansion

$$
\phi(z)=z+\alpha_{0}+\frac{\alpha_{1}}{z}+\cdots
$$

about the point at infinity. The Faber polynomial of degree $n$ is obtained by deleting all negative powers of $z$ from the corresponding Laurent expansion of $[\phi(z)]^{n}$. For the unit disc, the Faber polynomial of degree $n$ is $z^{n}$, and multiples of the Chebyshev polynomials of the first kind are the Faber polynomials for an ellipse with foci at $( \pm 1,0)$ and, in particular, for the real interval $[-1,1]$.

Properties of the Faber polynomials and Faber series are described in the books of Markushevich [24, v. 3, pp. 104-112], Smirnov and Lebedev [28, Chapter 2], Henrici [22, Chapter 18] and Gaier [17, pp. 42-57], and in a survey article by Curtiss [6]. The Faber polynomials are known explicitly only for a few types of domain. Those for certain lemniscates appear in [24], Elliott [15] computed the coefficients of some Faber polynomials for the semidisc $\{z:|z| \leq 1, \operatorname{Re} z \geq 0\}$ and for the square $\{z:|\operatorname{Re} z| \leq 1,|\operatorname{Im} z| \leq 1\}$, and Ellacott [10] expressed the Faber polynomials for a circular arc in terms of Chebyshev polynomials. Coleman and Smith [4] derived a recurrence relation for the coefficients of the Faber polynomials for circular sectors, and provided numerical values of those coefficients for polynomials of degree up to 15 for selected sectors [5]. Gatermann et al. [18] modified the algorithm of [4] to obtain a form involving only rational coefficients and therefore amenable to computer algebra systems; the algebraic forms in [19] allow the computation of the Faber polynomials of degree up to 20 for an arbitrary circular sector. In other cases, where explicit formulae were not available, numerical algorithms for conformal mapping have been used to generate Faber polynomials (see Ellacott [9], Starke and Varga [29] and Papamichael et al. [25]).

Virtues of suitably normalized Faber polynomials as residue polynomials for matrix iterative methods are described in [29]. In that case the Faber polynomials are required for some bounded region which contains the estimated locations of matrix eigenvalues produced by the Arnoldi method or otherwise. Since one is working with a rough prediction of the eigenvalue spectrum it is not necessary for the chosen enclosing region to bear any specific relation to the estimated eigenvalues. Professor G. Opfer suggested to one of the authors that an annular sector would be a useful general-purpose region which, by scaling and rotation, could be adjusted to enclose any estimated eigenvalue cluster bounded away from the origin. That suggestion motivated the work of the present paper, which provides explicit formulae for Faber polynomials for a sector of an annulus. Results for circular sectors are included as a special case. The main theoretical results are summarized in the following theorems.

Theorem 1. The complement of the unit disc $\{w:|w| \leq 1\}$ is mapped conformally onto the complement of the annular sector

$$
Q=\{z: R \leq|z| \leq 1, \theta \leq|\arg z| \leq \pi\}, \quad 0<\theta \leq \pi,
$$


by

$$
\psi(w)=-\exp \left[-\int_{a^{2}}^{-\zeta^{-1}} \frac{B(x)}{x A(x)} d x\right]
$$

where

$$
\zeta=\frac{1}{4 w}\left[\left(w^{2}+1\right)\left(a^{-2}-a^{2}\right)-2 w\left(a^{-2}+a^{2}\right)\right]
$$

and

$$
A(x)=\left[\left(x-a^{2}\right)\left(x-a^{-2}\right)\right]^{\frac{1}{2}}, \quad B(x)=\left[\left(x-b^{2}\right)\left(x-b^{-2}\right)\right]^{\frac{1}{2}} .
$$

The parameters $a$ and $b$ satisfy the equations

$$
\pi-\theta=\int_{a^{2}}^{b^{2}}\left[\frac{\left(b^{2}-x\right)\left(b^{-2}-x\right)}{\left(x-a^{2}\right)\left(a^{-2}-x\right)}\right]^{\frac{1}{2}} \frac{d x}{x}
$$

and

$$
\log R=-\int_{b^{2}}^{b^{-2}}\left[\frac{\left(x-b^{2}\right)\left(b^{-2}-x\right)}{\left(x-a^{2}\right)\left(a^{-2}-x\right)}\right]^{\frac{1}{2}} \frac{d x}{x} .
$$

Theorem 2. The transfinite diameter of the annular sector $Q$ defined in Theorem 1 is

$$
\rho=\frac{\left(1-a^{4}\right)}{4} \exp \left[\int_{0}^{a^{2}} \frac{a^{2}+a^{-2}-b^{2}-b^{-2}}{A(x)[A(x)+B(x)]} d x\right] .
$$

Theorem 3. The coefficients of the Laurent expansion

$$
\psi(w)=\rho\left(w+\beta_{0}+\beta_{1} w^{-1}+\cdots\right)
$$

of the function defined in Theorem 1 may be generated recursively. Given a and $b$, in the notation of Theorem 1 , let

$$
u=\frac{2 a^{2}\left(1+b^{4}\right)}{b^{2}\left(1-a^{4}\right)}, \quad s=2 \frac{1+a^{4}}{1-a^{4}} .
$$

Let $a_{i}=0$ for $i<0, a_{0}=1$ and, for $k \geq 0$,

$$
\begin{array}{rl}
(k+1) a_{k+1}=(2 k+1)(s-u) a_{k}-2 & k\left(s^{2}-s u-1\right) a_{k-1} \\
+ & (2 k-1)(s-u) a_{k-2}+(1-k) a_{k-3}
\end{array}
$$

and

$$
c_{k+1}=a_{k+1}-s a_{k}+a_{k-1} .
$$

Then $\beta_{0}=c_{1}, \beta_{1}=\frac{1}{2} c_{2}$ and, for $n \geq 2$,

$$
(n+1) \beta_{n}=c_{n+1}-\sum_{l=1}^{n-1} l c_{n-l} \beta_{l} .
$$

Theorem 4. The Faber polynomial of degree $n$ for the annular sector $Q$ defined in Theorem 1 is $F_{n}(z)=\rho^{n} \widetilde{F}_{n}(z)$, where $\rho$ is the transfinite diameter of $Q$. The scaled Faber polynomial may be written as $\widetilde{F}_{n}(\rho z)=z^{n}+\phi_{n-1}(z)$, and the 
ancillary polynomials $\left\{\phi_{n}\right\}$ are generated recursively, in terms of the Laurent coefficients of Theorem 3 , by the formula

$$
\phi_{n}(z)=\left(z-\beta_{0}\right) \phi_{n-1}(z)-\sum_{k=1}^{n-1} \beta_{k} \phi_{n-k-1}(z)-\sum_{k=0}^{n-1} \beta_{k} z^{n-k}-(1+n) \beta_{n} .
$$

Theorem 5. The Faber series for $z^{-1}$, expressed in terms of the scaled Faber polynomials and the notation of Theorem 1 , is

$$
\frac{1}{z}=-\frac{4 \rho a^{2}}{R\left(1-a^{4}\right)}\left[1+\sum_{n=1}^{\infty}\left(\frac{1-a^{2}}{1+a^{2}}\right)^{n} \widetilde{F}_{n}(z)\right] \text {. }
$$

Theorem 1 , which is proved in $\S 2$, provides an expression for the mapping function $\psi$, whose inverse is a multiple of the function $\phi$ of $(1.1)$; if $z=\psi(w)$, then $\phi(z)=\rho w$, where $\rho$ is given by Theorem 2. Section 3 establishes the formulae collected in Theorem 3, which allow the recursive evaluation of the Laurent coefficients essential for the computation of the Faber polynomials by the recurrence relation of Theorem 4. In addition to proving Theorem 4, $\S 4$ explores some of the properties of the ancillary polynomials $\left\{\phi_{n}\right\}$ and shows how explicit expressions for those polynomials may be obtained with the help of a computer algebra system such as REDUCE or Mathematica or Maple; the Supplement provides all that is required to determine the scaled Faber polynomials of degree $\leq 15$. The numerical evaluation of the parameters $a, b$, and $\rho$, for a given annular sector is discussed in $\S 5$.

Norms of Faber polynomials are of interest in connection with their use in approximations. Section 6 summarizes some results which are generalizations of those of Gatermann et al. [18] for circular sectors. As an example of a Faber series, the expansion of $1 / z$ is investigated in $\S 7$, where we find the simple formula stated in Theorem 5 .

\section{THE CONFORMAL MAPPING}

The aim of this section is to calculate an analytic function, $\psi(w)$, which maps the complement of the unit disc, $\Delta=\{w:|w| \leq 1\}$, conformally onto the exterior of the annular sector $Q=\{z: R \leq|z| \leq 1, \theta \leq|\arg z| \leq \pi\}$, where $0<\theta \leq \pi$ (see Figure 1). There is no loss of generality in this choice since rotations and magnifications allow us to apply the results to any annular sector. For example, to work with the annular sector $\{z: R \leq|z| \leq 1,|\arg z| \leq \theta\}$ we make the transformations $z \rightarrow-z$ and $w \rightarrow-w$, the latter being required to maintain the form (1.1).

The domain obtained by cutting $\mathbb{C} \backslash Q$ along its intersection with the negative real axis is mapped conformally onto the shaded domain $E$ of Figure 2 by the function $z \rightarrow \log z$, where the principal value of the logarithm is taken. The domain $E$ is the interior of an infinite polygon with finite vertices at the points $\log R \pm i \pi, \log R \pm i \theta, \pm i \theta$, and $\pm i \pi$. Conversely, the function $z \rightarrow e^{z}$ maps $E$ conformally onto the cut version of $\mathbb{C} \backslash Q$, and the infinite edges of the boundary of $E$ are mapped onto the cut.

A Schwarz-Christoffel transformation may be used to map the upper halfplane $\Pi=\{v: \operatorname{Im} v>0\}$ conformally onto the domain $E$ in such a way that the real axis is mapped onto the polygonal boundary in Figure 2 and the finite 


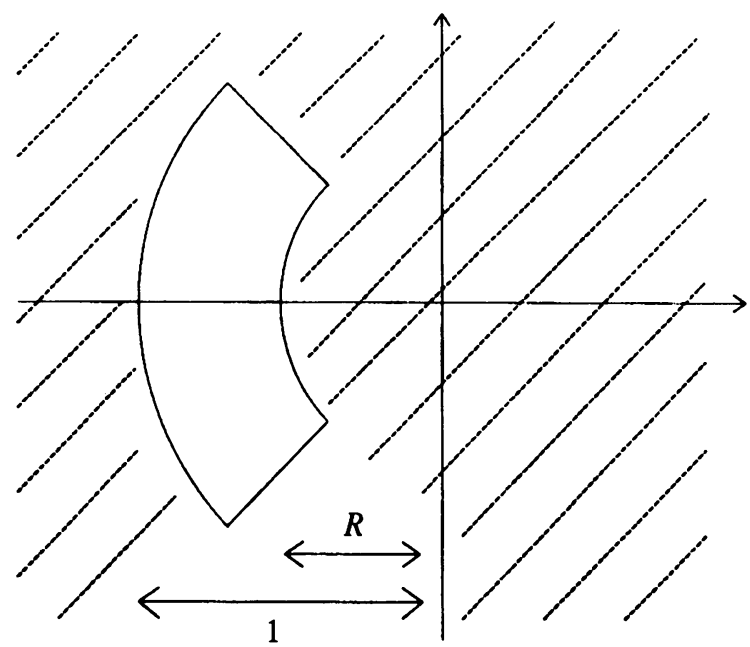

Figure 1. The annular sector $Q$

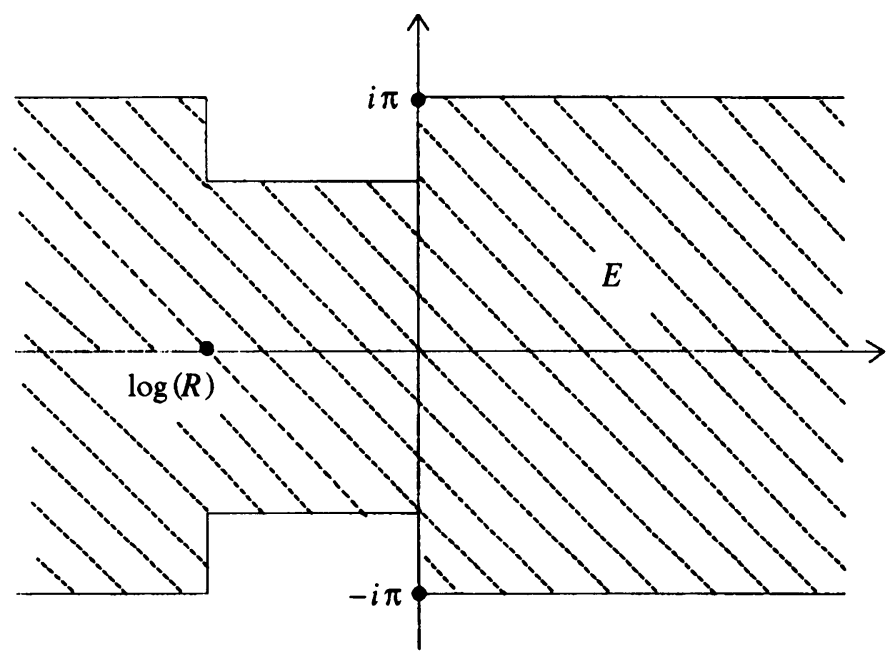

FIGURE 2

vertices of that polygon are the images of the points $\pm a^{-1}, \pm b^{-1}, \pm b$, and $\pm a$, with $0<a \leq b \leq 1$. The Schwarz-Christoffel map has the form

$$
u(v)=u(a)+K \int_{a}^{v}\left[\frac{(w-b)(w+b)\left(w-b^{-1}\right)\left(w+b^{-1}\right)}{(w-a)(w+a)\left(w-a^{-1}\right)\left(w+a^{-1}\right)}\right]^{\frac{1}{2}} \frac{d w}{w}
$$

where $a, b$, and $K$ are constants to be determined.

Let

$$
A(x)=\left[\left(x-a^{2}\right)\left(x-a^{-2}\right)\right]^{\frac{1}{2}}, \quad B(x)=\left[\left(x-b^{2}\right)\left(x-b^{-2}\right)\right]^{\frac{1}{2}} .
$$


Then

$$
\begin{aligned}
u(v)-u(a) & =K \int_{a}^{v} \frac{B\left(w^{2}\right)}{w A\left(w^{2}\right)} d w \\
& =K \int_{a}^{v} \frac{B\left(w^{2}\right)-A\left(w^{2}\right)}{w A\left(w^{2}\right)} d w+K \log \left(\frac{v}{a}\right) \\
& =K \int_{a^{2}}^{v^{2}} \frac{\cosh \alpha-\cosh \beta}{A(x)[B(x)+A(x)]} d x+K \log \left(\frac{v}{a}\right),
\end{aligned}
$$

where we have written

$$
a^{-2}=e^{\alpha} \text { and } b^{-2}=e^{\beta}
$$

To determine the constant $K$, we note that the positive real axis is mapped onto the upper boundary of the polygon in Figure 2 , and the lower boundary is the image of the negative real axis. Therefore, for all $v \in(0, a)$,

$$
2 \pi i=u(v)-u(-v)=K\left[\log \left(\frac{v}{a}\right)-\log \left(-\frac{v}{a}\right)\right]=-K \pi i
$$

from which $K=-2$. Furthermore, we require

$$
u(a)=i \pi
$$

and (2.2) becomes

$$
u(v)=i \pi-\int_{a^{2}}^{v^{2}} \frac{B(x)}{x A(x)} d x .
$$

If we assume that $a$ and $b$ can be determined for any given annular sector (see $\S 2.1$ ), the rest of the argument is similar to that of Coleman and Smith [4]. A composition of the Joukowski function $w \rightarrow \frac{1}{2}\left(w^{-1}+w\right)$ and a linear transformation gives

$$
\zeta=\frac{1}{2 w}\left[\left(w^{2}+1\right) \sinh \alpha-2 w \cosh \alpha\right],
$$

which maps $\mathbb{C} \backslash \Delta$, the complement of the unit disc $\Delta$, conformally onto the slit plane $\mathbb{C} \backslash J$, where $J$ is the interval $\left[-a^{-2},-a^{2}\right]$. Let $L$ denote the interval $(-\infty, 0]$ of the real axis. Then the function $\zeta \rightarrow i \zeta^{-\frac{1}{2}}$ maps the cut plane $\mathbb{C} \backslash L$ conformally onto the upper half-plane $\Pi$.

By composition of the mappings described here we obtain

$$
z=-\exp \left[-\int_{a^{2}}^{-\zeta^{-1}} \frac{B(x)}{x A(x)} d x\right],
$$

which maps $\mathbb{C} \backslash L$ conformally onto the cut version of $\mathbb{C} \backslash Q$. At the end of $\S 2.2$ it will be shown that the cut introduced in $\mathbb{C} \backslash Q$ may be removed and that $\mathbb{C} \backslash L$ is then replaced by $\mathbb{C} \backslash J$.

2.1. Defining equations for $a$ and $b$. The symmetries inherent in our choice of the preimages of the vertices of the polygon, under the Schwarz-Christoffel map, preserve the relationships, which are evident in Figure 2 , between the lengths of the edges. The two distinct lengths which arise are

$$
\pi-\theta=i[u(b)-u(a)]
$$


and

$$
-\log R=u(b)-u\left(b^{-1}\right) .
$$

These equations, which may be written as

$$
\pi-\theta=\int_{a^{2}}^{b^{2}}\left[\frac{\left(b^{2}-x\right)\left(b^{-2}-x\right)}{\left(x-a^{2}\right)\left(a^{-2}-x\right)}\right]^{\frac{1}{2}} \frac{d x}{x}
$$

and

$$
\log R=-\int_{b^{2}}^{b^{-2}}\left[\frac{\left(x-b^{2}\right)\left(b^{-2}-x\right)}{\left(x-a^{2}\right)\left(a^{-2}-x\right)}\right]^{\frac{1}{2}} \frac{d x}{x},
$$

uniquely determine $\theta$ and $R$ for any given $a$ and $b$ such that $0<a \leq b \leq 1$. Furthermore, as the geometrical interpretation in Figure 1 requires, $0<\theta \leq \pi$. Since the integrand in (2.7) is nonnegative, the right-hand inequality is true and, since $b^{2}+b^{-2}$ is a decreasing function of $b$,

$$
\pi-\theta \leq \int_{a^{2}}^{1} \frac{1-x}{\left[\left(x-a^{2}\right)\left(a^{-2}-x\right)\right]^{\frac{1}{2}}} \frac{d x}{x}=\pi-2 \sin ^{-1}\left(\frac{2 a}{a^{2}+1}\right) ;
$$

in particular, $\theta>0$. Equation (2.8) may be expressed in a form which is more useful for numerical computation, by regarding its right-hand side as a sum of integrals on $\left[b^{2}, 1\right]$ and $\left[1, b^{-2}\right]$. Making the transformation $x \rightarrow x^{-1}$ in the second integral, we obtain

$$
\log R=-2 \int_{b^{2}}^{1}\left[\frac{\left(x-b^{2}\right)\left(b^{-2}-x\right)}{\left(x-a^{2}\right)\left(a^{-2}-x\right)}\right]^{\frac{1}{2}} \frac{d x}{x} .
$$

The integrals in (2.7) and (2.8) may be expressed in terms of elliptic integrals of the first and third kinds. In a standard notation [27] we find

$$
\begin{aligned}
\pi-\theta=\frac{2 a b}{1-a^{2} b^{2}}[C F & \left(\frac{\pi}{2}, k\right)+\left(a^{2}-a^{-2}\right) \\
& \left.\times\left\{\Pi\left(\frac{\pi}{2},-a^{2} k, k\right)-\Pi\left(\frac{\pi}{2},-\frac{k}{a^{2}}, k\right)\right\}\right]
\end{aligned}
$$

and

$$
\begin{aligned}
\log R=\frac{-2 a b}{1-a^{2} b^{2}}[C F & \left(\frac{\pi}{2}, k_{1}\right)+\left(b^{2}-a^{2}\right) \\
\times & \left.\left\{\Pi\left(\frac{\pi}{2}, k_{2}, k_{1}\right)-\frac{1}{a^{2} b^{2}} \Pi\left(\frac{\pi}{2}, \frac{a^{2}}{b^{2}} k_{2}, k_{1}\right)\right\}\right],
\end{aligned}
$$

where

$$
k=\frac{b^{2}-a^{2}}{1-a^{2} b^{2}}, \quad k_{1}=\frac{\left(1-a^{4}\right)^{\frac{1}{2}}\left(1-b^{4}\right)^{\frac{1}{2}}}{\left(1-a^{2} b^{2}\right)}, \quad k_{2}=\frac{\left(1-b^{4}\right)}{\left(1-a^{2} b^{2}\right)},
$$

and

$$
C=2(\cosh \alpha-\cosh \beta) \text {. }
$$

2.2. Formulae for $\psi(w)$. The mapping function $\psi$ may be written in several different ways, which we list here for later reference. 
By using the change of variable $x \rightarrow x^{-1}$ in the first formula quoted, by taking $a^{-1}$ as reference point instead of $a$ as in (2.2), and by using the substitution $x \rightarrow x^{-1}$ again, we obtain

$$
\begin{aligned}
z=\psi(w) & =-\exp \left[-\int_{a^{2}}^{-\zeta^{-1}} \frac{B(x)}{x A(x)} d x\right] \\
& =-\exp \left[\int_{a^{-2}}^{-\zeta} \frac{B(x)}{x A(x)} d x\right] \\
& =-R \exp \left[\int_{a^{2}}^{-\zeta} \frac{B(x)}{x A(x)} d x\right] \\
& =-R \exp \left[-\int_{a^{-2}}^{-\zeta^{-1}} \frac{B(x)}{x A(x)} d x\right],
\end{aligned}
$$

where

$$
\zeta=\frac{1}{2 w}\left[\left(w^{2}+1\right) \sinh \alpha-2 w \cosh \alpha\right] .
$$

In some cases, particularly if the integration interval may include the origin, it is preferable to remove the logarithmic term from the integral, as in the derivation of (2.3). Corresponding to the formulae (2.12), we have, with $C$ as in $(2.11)$,

$$
z=a^{2} \zeta \exp \left[-\int_{a^{2}}^{-\zeta^{-1}} \frac{C}{A(x)[A(x)+B(x)]} d x\right]
$$

$$
\begin{aligned}
& =a^{2} \zeta \exp \left[\int_{a^{-2}}^{-\zeta} \frac{C}{A(x)[A(x)+B(x)]} d x\right] \\
& =\frac{R \zeta}{a^{2}} \exp \left[\int_{a^{2}}^{-\zeta} \frac{C}{A(x)[A(x)+B(x)]} d x\right] \\
& =\frac{R \zeta}{a^{2}} \exp \left[-\int_{a^{-2}}^{-\zeta^{-1}} \frac{C}{A(x)[A(x)+B(x)]} d x\right] .
\end{aligned}
$$

Other forms, which do not involve the intermediate variable $\zeta$, may be derived from (2.12). For example, the change of variable

$$
x=\frac{-1}{2 \mu}\left[\left(\mu^{2}+1\right) \sinh \alpha-2 \mu \cosh \alpha\right]
$$

applied to (2.12b) gives, after some algebra,

$$
z=\psi(w)=-\exp \left[\int_{-1}^{w} \frac{\left(\mu^{2}-2 t \mu+1\right)^{\frac{1}{2}}\left(\mu^{2}-2 \tau \mu+1\right)^{\frac{1}{2}}}{\mu\left(\mu^{2}-2 \mu \operatorname{coth} \alpha+1\right)} d \mu\right],
$$

where

$$
t=\frac{\cosh \alpha-b^{2}}{\sinh \alpha}, \quad \tau=\frac{\cosh \alpha-b^{-2}}{\sinh \alpha}
$$


By combining these definitions with (2.4), it is easily seen that $|t| \leq 1$ and $|\tau| \leq 1$ for $0<a \leq b \leq 1$.

To complete the proof that the function $\psi$ provides the required mapping from $\mathbb{C} \backslash \Delta$ to $\mathbb{C} \backslash J$, it is necessary to show that we may remove the cuts introduced in constructing $\psi$. Let

$$
\begin{aligned}
\chi(\zeta) & =a^{2} \exp \left[\int_{a^{-2}}^{-\zeta} \frac{C}{A(x)[A(x)+B(x)]} d x\right] \\
& =a^{2} \exp \left[-\int_{a^{2}}^{-\zeta^{-1}} \frac{C}{A(x)[A(x)+B(x)]} d x\right] .
\end{aligned}
$$

The denominator of the integrand is a single-valued analytic function in $\mathbb{C} \backslash J$, the region of the complex plane exterior to the slit $J$, and it does not vanish in that region. Integration gives a single-valued analytic function in $\mathbb{C} \backslash J$ and the identity of the two integrals shows that it remains finite as $\zeta \rightarrow \infty$. It follows that $\chi(\zeta)$ is a single-valued analytic function in $\mathbb{C} \backslash J$ and, consequently, $\zeta \chi(\zeta)$ is also single-valued in $\mathbb{C} \backslash J$. Since the part of the interval $L$ which lies in $\mathbb{C} \backslash J$ is mapped onto that part of the negative real axis which lies in $\mathbb{C} \backslash Q$, the function $\psi$ given by (2.12) and (2.13) maps $\mathbb{C} \backslash \Delta$ conformally onto $\mathbb{C} \backslash Q$.

2.3. The transfinite diameter. Given the mapping $z=\psi(w)$, we now define

$$
\rho=\lim _{w \rightarrow \infty} \frac{\psi(w)}{w}
$$

and $\phi(z)=\rho \psi^{-1}(z)$, where $\psi^{-1}$ denotes the inverse mapping of $\psi$. Then $\phi(z)$ will map $\mathbb{C} \backslash Q$ conformally onto the complement of the disc $\{w:|w| \leq \rho\}$, so the number $\rho$ is the transfinite diameter of the annular sector $Q$. As $w \rightarrow$ $\infty$,

$$
\zeta=\frac{1}{2} w \sinh \alpha\left[1+O\left(\frac{1}{w}\right)\right] .
$$

Therefore, from (2.13a) and (2.16),

$$
\rho=\frac{\left(1-a^{4}\right)}{4} \exp \left[\int_{0}^{a^{2}} \frac{C}{A(x)[A(x)+B(x)]} d x\right] .
$$

2.4. Special cases. There are three special cases which provide useful checks on the mapping, the formula for the transfinite diameter and the Faber polynomials themselves.

Case (i): $b=a$. A real interval

When $b=a$, the equations (2.7) and (2.8) give $\theta=\pi$ and $R=a^{4}$. Consequently, the annular sector $Q$ becomes the interval $[-1,-R]$ of the real axis.

From (2.3) it is evident that

$$
u(v)=i \pi-2 \log \left(\frac{v}{a}\right)
$$

when $b=a$. Therefore,

$$
z=e^{u}=a^{2} \zeta=\frac{1}{4}\left(w+\frac{1}{w}\right)(1-R)-\frac{1}{2} w(1+R),
$$


which correctly maps $\mathbb{C} \backslash \Delta$ onto the complement of the real interval $[-1,-R]$.

When $b=a$, the integrand in (2.17) vanishes, so

$$
\rho=\frac{\left(1-a^{4}\right)}{4}=\frac{1}{4}(1-R) .
$$

Case (ii): $b=1$. A circular arc

When $b=1$, the integral in (2.8) vanishes to give $R=1$, and (2.7) gives (see $(2.9))$

$$
\theta=2 \sin ^{-1}\left(\frac{2 a}{a^{2}+1}\right) .
$$

The annular sector $Q$ then degenerates to an arc of the unit circle $|z|=1$, of half-angle $\pi-\theta$.

In this case, $\tau=t$ in (2.14), and

$$
\begin{aligned}
\psi(w) & =-\exp \left[\int_{-1}^{w} \frac{\mu^{2}-2 t \mu+1}{\mu\left(\mu^{2}-2 \mu \operatorname{coth} \alpha+1\right)} d \mu\right] \\
& =w \exp \left[\frac{2}{\sinh \alpha} \int_{-1}^{w} \frac{d \mu}{\mu^{2}-2 \mu \operatorname{coth} \alpha+1}\right] .
\end{aligned}
$$

The remaining integral is elementary, and the result is

$$
z=\psi(w)=\frac{w\left(w \tanh \frac{\alpha}{2}-1\right)}{w-\tanh \frac{\alpha}{2}}
$$

which correctly maps $\mathbb{C} \backslash \Delta$ onto the complement of the circular arc

$$
\{z:|z|=1, \theta \leq|\arg z| \leq \pi\},
$$

where $\theta$ is given by equation (2.18) .

From the expression (2.19) for $\psi(w)$, the transfinite diameter of the arc is,

$$
\rho=\tanh \frac{\alpha}{2}=\left(\frac{1-a^{2}}{1+a^{2}}\right)=\cos \frac{\theta}{2},
$$

which, with allowance for the difference in notation, agrees with Ellacott [10]. The formula (2.17) similarly gives

$$
\rho=\frac{\left(1-a^{4}\right)}{4} \exp \left[2 \log \left(\frac{2}{a^{2}+1}\right)\right]=\left(\frac{1-a^{2}}{1+a^{2}}\right) .
$$

Case (iii): $a \rightarrow 0$ and $b \rightarrow 0$. $A$ circular sector

In the integrand of (2.7), $x<<b^{-2} \leq a^{-2}$ when $0<a \leq b<<1$. Expanding $\left(b^{-2}-x\right)^{\frac{1}{2}}\left(a^{-2}-x\right)^{-\frac{1}{2}}$ in terms of $a$ and $b$, and evaluating the integrals, we obtain

so when $a \rightarrow 0$ and $b \rightarrow 0$, we have

$$
\pi-\theta=\left(\pi-\frac{a \pi}{b}\right)\left[1+O\left(a^{2}\right)+O\left(b^{2}\right)\right]
$$

$$
\theta \rightarrow \frac{\pi a}{b}
$$

Similarly, from (2.10),

$$
\log R=-\frac{2 a}{b}\left[\int_{b^{2}}^{1} \frac{\left(x-b^{2}\right)^{\frac{1}{2}}}{x\left(x-a^{2}\right)^{\frac{1}{2}}} d x+O\left(a^{2}\right)+O\left(b^{2}\right)\right] .
$$


The substitution $y^{2}=\left(x-b^{2}\right) /\left(x-a^{2}\right)$ allows us to evaluate the integral as

$$
\log \left(\frac{1+y_{0}}{1-y_{0}}\right)+\frac{b}{a} \log \left(\frac{b-a y_{0}}{b+a y_{0}}\right)
$$

with $y_{0}=\left(1-b^{2}\right)^{\frac{1}{2}}\left(1-a^{2}\right)^{-\frac{1}{2}}$. Since further expansion shows that $R \rightarrow 0$ as $a$ and $b$ both tend to zero, the annular sector $Q$ tends to the circular sector

$$
\{z:|z| \leq 1, \theta \leq|\arg z| \leq \pi\}
$$

where $\theta=\pi a / b$; our results in that limit should agree with those of Coleman and Smith [4] and of Gatermann et al. [18].

To find the transfinite diameter in the required limit, we write $a=\lambda b$ in equation (2.17) and make the substitution $x=t b^{2}$ to obtain

$$
\rho=\frac{\left(1-\lambda^{4} b^{4}\right)}{4} \exp [I(\lambda, b)]
$$

where

$$
I(\lambda, b)=\int_{0}^{\lambda^{2}} \frac{\left[b^{4}\left(\lambda^{2}-1\right)+\lambda^{-2}-1\right] d t}{\left(\lambda^{2}-t\right)^{\frac{1}{2}}\left(\lambda^{-2}-t b^{4}\right)^{\frac{1}{2}}\left[\left(\lambda^{2}-t\right)^{\frac{1}{2}}\left(\lambda^{-2}-t b^{4}\right)^{\frac{1}{2}}+(1-t)^{\frac{1}{2}}\left(1-b^{4} t\right)^{\frac{1}{2}}\right]} .
$$

In the limit as $b \rightarrow 0$ this reduces to

$$
\rho=\frac{1}{4} \exp \left[\int_{0}^{\lambda^{2}}\left\{\frac{\lambda(1-t)^{\frac{1}{2}}}{\left(\lambda^{2}-t\right)^{\frac{1}{2}}}-1\right\} \frac{d t}{t}\right] .
$$

The substitution $y^{2}=(1-t) /\left(\lambda^{2}-t\right)$ and use of partial fractions converts the integral to a sum of elementary integrals, and we find

$$
\rho=\frac{1}{(1-\lambda)^{1-\lambda}(1+\lambda)^{1+\lambda}},
$$

and in this limit $\lambda=\theta / \pi$. Theorem 2 of Coleman and Smith [4] shows that this is the transfinite diameter for a sector of the unit disc of half-angle $\pi-\theta$.

Similar reasoning applied to the integral in (2.13a) allows us to compute $\psi_{0}$, the limit of the mapping function $\psi$ as $a$ and $b$ both tend to 0 . Noting that

$$
\zeta=\frac{1}{4 a^{2} w}\left[(w-1)^{2}+O\left(a^{4}\right)\right],
$$

as $a \rightarrow 0$, we find

$$
\psi_{0}(w)=\frac{(w-1)^{2}}{4 w} \exp \left[\int_{-x_{0}}^{\lambda^{2}}\left\{\frac{\lambda(1-t)^{\frac{1}{2}}}{\left(\lambda^{2}-t\right)^{\frac{1}{2}}}-1\right\} \frac{d t}{t}\right],
$$

where $x_{0}=4 \lambda^{2} w(w-1)^{-2}$.

To obtain the configuration chosen by Coleman and Smith [4], it is necessary to carry out the transformations $z \rightarrow-z$ and $w \rightarrow-w$. In the notation of [4], modified to avoid conflicting uses of $a$,

$$
u(-w)=\frac{w+1}{2 a_{c} w^{\frac{1}{2}}}, \quad a_{c}^{2}=1-\lambda^{2},
$$

when the correct branch of the square root function is chosen. Straightforward algebraic manipulation then shows that $-\psi_{0}(-w)$ is the mapping function in 
Theorem 1 of Coleman and Smith [4], thus confirming that the map derived here correctly reduces to that for a circular sector in the limit as $a \rightarrow 0$ and $b \rightarrow 0$.

3. The LAURent EXPANSION of $\psi(w)$ AbOUt the POINT AT INFINITY

The function $\psi(w)$ has a Laurent expansion of the form

$$
\psi(w)=\rho\left(w+\beta_{0}+\beta_{1} w^{-1}+\cdots\right)
$$

about the point at infinity. The coefficients of this expansion are required in a recurrence relation used to generate the Faber polynomials.

Differentiation of (2.14) gives

$$
\frac{d \psi}{d w}=\psi(w) \frac{M(w, t) M(w, \tau)}{w\left(w^{2}-2 w \operatorname{coth} \alpha+1\right)},
$$

where

$$
M(w, y)=\left(w^{2}-2 y w+1\right)^{\frac{1}{2}} .
$$

If we now let $w=\xi^{-1}$ and $\Psi(\xi)=\psi(w)$, equation (3.2) may be rewritten as

$$
\Psi(\xi)=-\frac{\xi\left(\xi^{2}-2 \xi \operatorname{coth} \alpha+1\right)}{M(\xi, t) M(\xi, \tau)} \frac{d \Psi(\xi)}{d \xi} .
$$

Since $|t| \leq 1$ and $|\tau| \leq 1$, there is a convergent expansion of the form

$$
[M(\xi, t) M(\xi, \tau)]^{-1}=\sum_{k=0}^{\infty} a_{k} \xi^{k}
$$

for $|\xi|<1$. Substitution in (3.3) gives

$$
\Psi(\xi)=\left(\sum_{k=0}^{\infty} c_{k} \xi^{k}\right)\left(-\xi \frac{d \Psi}{d \xi}\right),
$$

where

$$
c_{k}=a_{k-2}-2 \operatorname{coth} \alpha a_{k-1}+a_{k}, \quad k \geq 0,
$$

with $a_{-2}=a_{-1}=0$. Also, from (3.1),

$$
\Psi(\xi)=\rho\left(\frac{1}{\xi}+\beta_{0}+\beta_{1} \xi+\cdots\right)
$$

for $|\xi|<1$. We may substitute this in (3.5) and equate coefficients of the powers of $\xi$ on both sides to obtain $\beta_{0}=c_{1}, \beta_{1}=\frac{1}{2} c_{2}$ and, for $n \geq 2$,

$$
(1+n) \beta_{n}=c_{n+1}-\sum_{i=1}^{n-1} i c_{n-i} \beta_{i} .
$$

The recurrence relation in (3.7) and the definition (3.6) allow us to generate $\beta_{0}, \beta_{1}, \ldots, \beta_{n}$, for a given positive integer $n$, when $a_{k}$ is known for $k=$ $0,1, \ldots, n+1$. In the case of circular sectors, Coleman and Smith [4] found a very simple expression for the corresponding coefficients in terms of Legendre 
polynomials. A similar approach can be used here; noting that $M(w, y)$ is the reciprocal of a generating function for the Legendre polynomials, we find

$$
a_{k}=\sum_{l=0}^{k} P_{l}(t) P_{k-l}(\tau)
$$

where $P_{n}$ is the Legendre polynomial of degree $n$. This formula has the computational disadvantage that as $k$ increases an increasing number of Legendre polynomials must be evaluated and stored. For this reason we have derived a five-term recurrence relation from which $\left\{a_{k}\right\}$ may be computed directly. Let $f(t, \tau, \xi)$ be the function in (3.4). Then, by differentiation and rearrangement,

$$
\begin{aligned}
{[t+\tau-2 \xi} & \left.(1+2 t \tau)+3(t+\tau) \xi^{2}-2 \xi^{3}\right] f(t, \tau, \xi) \\
& =\left(1-2 t \xi+\xi^{2}\right)\left(1-2 \tau \xi+\xi^{2}\right) \frac{\partial f(t, \tau, \xi)}{\partial \xi} .
\end{aligned}
$$

Equating the coefficients of the powers of $\xi$ on both sides of the equation, we obtain

$$
\begin{aligned}
(k+1) a_{k+1}=(2 k+1)(t+\tau) a_{k}-2 k(1+2 t \tau) a_{k-1} \\
+(2 k-1)(t+\tau) a_{k-2}+(1-k) a_{k-3}
\end{aligned}
$$

for $k \geq 0$, with $a_{0}=1$ and $a_{i}=0$ for $i<0$. With

$$
s=2 \frac{\cosh \alpha}{\sinh \alpha} \text { and } u=2 \frac{\cosh \beta}{\sinh \alpha}
$$

this becomes

$$
\begin{aligned}
(k+1) a_{k+1}=(2 k+1)(s-u) a_{k}-2 k\left(s^{2}-s u-1\right) a_{k-1} \\
+(2 k-1)(s-u) a_{k-2}+(1-k) a_{k-3} .
\end{aligned}
$$

\section{The FABER POLYNOMIALS}

The Faber polynomials, $F_{n}(z)$, satisfy the recurrence relation

$$
F_{n+1}(z)=\left(z-b_{0}\right) F_{n}(z)-\sum_{k=1}^{n-1} b_{k} F_{n-k}(z)-(1+n) b_{n}, \quad n>0,
$$

where $b_{k}=\beta_{k} \rho^{k+1}, \rho$ is the transfinite diameter of the region, and the $\beta_{k}$ are generated from the recurrence relations above. Following Gatermann et al. [18], we introduce the scaled Faber polynomials

$$
\widetilde{F}_{n}(z)=F_{n}(z) \rho^{-n}=\Phi_{n}\left(\frac{z}{\rho}\right)
$$

and let

$$
\Phi_{n}(z)=z^{n}+\phi_{n-1}(z),
$$

where $\phi_{n-1}$ is a polynomial of degree $n-1$ for $n \geq 1$, and $\phi_{-1}(z)=0$.

Substitution in (4.1) gives the recurrence relation

$$
\phi_{n}(z)=\left(z-\beta_{0}\right) \phi_{n-1}(z)-\sum_{k=1}^{n-1} \beta_{k} \phi_{n-k-1}(z)-\sum_{k=0}^{n-1} \beta_{k} z^{n-k}-(1+n) \beta_{n} .
$$


Our notation differs slightly from that used by Gatermann et al. [18], in their work on circular sectors, because no factor analogous to their $1-c$ is evident, except in the limit as $a \rightarrow 0$ and $b \rightarrow 0$, when the annular sector tends to a sector of the unit disc. Given the Schwarz-Christoffel parameters $a$ and $b$ corresponding to a particular annular sector, the Faber polynomials of degree up to $n_{\max }$ may be computed by the following algorithm.

\section{Algorithm.}

$$
\begin{aligned}
& s=2\left(1+a^{4}\right) /\left(1-a^{4}\right) ; u=2 a^{2} b^{-2}\left(1+b^{4}\right) /\left(1-a^{4}\right) ; \\
& a_{-3}=a_{-2}=a_{-1}=0 ; a_{0}=1 ; a_{1}=s-u ; \\
& c_{1}=a_{1}-s ; \beta_{0}=c_{1} ; \phi_{0}=-\beta_{0} ; \\
& F_{0}(z)=1 ; F_{1}(z)=z+\rho \phi_{0} . \\
& \text { For } \left.n=1, n_{\max }-1 \quad+(2 n-1)(s-u) a_{n-2}+(1-n) a_{n-3}\right] /(n+1) ; \\
& \quad a_{n+1}=\left[(2 n+1)(s-u) a_{n}-2 n\left(s^{2}-s u-1\right) a_{n-1}\right. \\
& \left.\quad c_{n+1}=a_{n+1}-s a_{n}+a_{n-1} ; \quad \quad+\beta_{l}\right) \\
& \quad \beta_{n}=\left(c_{n+1}-\sum_{l=1}^{n-1} l c_{n-l} \beta_{l}\right) /(n+1) ; \\
& \quad \phi_{n}=\left(z-\beta_{0}\right) \phi_{n-1}-\sum_{k=1}^{n-1} \beta_{k}\left(\phi_{n-k-1}+z^{n-k}\right)-(1+n) \beta_{n}-\beta_{0} z^{n} ; \\
& \quad \Phi_{n+1}=z^{n+1}+\phi_{n} ; \quad F_{n+1}(z)=\rho^{n+1} \Phi_{n+1}\left(\frac{z}{\rho}\right) .
\end{aligned}
$$

end.

Example. With $n_{\max }=2$ we obtain

$$
\begin{aligned}
& a_{0}=1 ; \quad a_{1}=s-u ; \quad c_{1}=-u ; \quad \beta_{0}=-u ; \quad \phi_{0}=u ; \\
& a_{2}=\left(s^{2}-4 s u+3 u^{2}+2\right) / 2 ; \quad c_{2}=\left(-s^{2}-2 s u+3 u^{2}+4\right) / 2 ; \\
& \beta_{1}=\left(-s^{2}-2 s u+3 u^{2}+4\right) / 4 ; \quad \phi_{1}=2 u z+\left(s^{2}+2 s u-u^{2}-4\right) / 2 ; \\
& a_{3}=\left(-s^{3}-3 s^{2} u+9 s u^{2}-5 u^{3}+8 s-8 u\right) / 2 ; \\
& c_{3}=\left(-2 s^{3}+s^{2} u+6 s u^{2}-5 u^{3}+8 s-10 u\right) / 2 ; \\
& \beta_{2}=\left(-4 s^{3}+s^{2} u+10 s u^{2}-7 u^{3}+16 s-16 u\right) / 12 ; \\
& \phi_{2}=3 u z^{2}+\left(3 s^{2}+6 s u+3 u^{2}-12\right) z / 4+\left(2 s^{3}+s^{2} u-2 s u^{2}+u^{3}+2 u-8 s\right) / 2 . \\
& \text { Then } \Phi_{1}(z)=z+u, \quad \Phi_{2}(z)=z^{2}+\phi_{1}(z), \quad \Phi_{3}(z)=z^{3}+\phi_{2}(z) .
\end{aligned}
$$

In the limit as $a \rightarrow 0$ and $b \rightarrow 0$, when the annular sector becomes a sector of the unit disc, we have that $s \rightarrow 2$ and, from (2.20),

$$
u \rightarrow 2\left(\frac{\theta}{\pi}\right)^{2}=2(1-c)
$$

where the parameter $c$ is as used by Gatermann et al. [18]. Making the changes described at the end of $\S 2.4$ to obtain the appropriate sector, we find, for example,

$$
\begin{aligned}
& \Phi_{1}(z)=z-2(1-c), \\
& \Phi_{2}(z)=z^{2}+(1-c)[-4 z+2+2 c], \\
& \Phi_{3}(z)=z^{3}+(1-c)\left[-6 z^{2}+(9-3 c) z+-2-4 c\right],
\end{aligned}
$$

which agree with Gatermann et al. [18]. 
4.1. The coefficients of $\phi_{n}(z)$. Letting

$$
\phi_{n}(z)=\sum_{j=0}^{n} p_{n, j} z^{n-j}
$$

in the recurrence relation (4.4), we obtain, after some algebra,

$$
\begin{gathered}
p_{n, 0}=p_{n-1,0}-\beta_{0}=(n+1) u \\
p_{n, n}=-\sum_{k=1}^{n} \beta_{n-k} p_{k-1, k-1}-(1+n) \beta_{n}
\end{gathered}
$$

and, for $i=1, \ldots, n-1$,

$$
p_{n, n-i}=p_{n-1, n-i}-\sum_{k=i+1}^{n} \beta_{n-k} p_{k-1, k-1-i}-\beta_{n-i} .
$$

In the interest of brevity we shall use the term $A$-polynomial to describe a polynomial in two variables, $s$ and $u$, which is invariant or changes sign, under the planar antipodal map $(s, u) \rightarrow(-s,-u)$, according as the degree of the polynomial is even or odd; in other words, such a polynomial of even (odd) degree contains only terms of the form $s^{i} u^{j}$ where $i+j$ is even (odd).

Theorem 6. The coefficient $p_{n, j}$, for $n=0,1, \ldots$ and $j=0, \ldots, n$, is an A-polynomial of degree $j+1$ in $s$ and $u$.

Proof. An induction argument applied to the recurrence relation (3.8) shows that $a_{k}$ is a polynomial in $s$ and $u$ of degree $k$. Furthermore, since $s-u$ and $s^{2}-s u-1$ are $A$-polynomials of degree 1 and 2 , respectively, the hypothesis that $a_{k}$ is an $A$-polynomial of degree $k$ for $k=0, \ldots, n$ leads to the conclusion that the same property holds for $k=n+1$; the induction hypothesis is readily confirmed for $n=1$. It then follows from (3.6) that $c_{n}$ is an $A$-polynomial of degree $n$, and an induction argument applied to equation (3.7) shows that $\beta_{n}$ is an $A$-polynomial of degree $n+1$.

Turning now to the equations (4.6), we assume, as an induction hypothesis, that for each $n$ the coefficient $p_{n, j}$ is an $A$-polynomial of degree $j+1$. Then each term on the right-hand side of (4.6c) is an $A$-polynomial of degree $n-i+1$, and (4.6b) gives the corresponding result for $i=0$; finally (4.6a) shows that $p_{n, 0}$ is an $A$-polynomial of degree 1 . Clearly the hypothesis is true for $n=1$.

In view of Theorem 6 , we may write

$$
\begin{aligned}
& p_{n-1,0}=\gamma_{n 1} u, \\
& p_{n-1,1}=\gamma_{n 2}+\gamma_{n 3} s^{2}+\gamma_{n 4} s u+\gamma_{n 5} u^{2}, \\
& p_{n-1,2}=\gamma_{n 6} s+\gamma_{n 7} u+\gamma_{n 8} s^{3}+\gamma_{n 9} s^{2} u+\gamma_{n 10} s u^{2}+\gamma_{n 11} u^{3},
\end{aligned}
$$

etc. In keeping with the notation of Gatermann et al. [18], we regard the coefficients $\gamma_{n k}$ as the elements of the $n$th row of a matrix

$$
\Gamma=\left(\gamma_{n k}\right), \quad n=1,2, \ldots ; k=1,2, \ldots, m(n),
$$

where $m(n)$ is the number of terms in the $n$th row of $\Gamma$. 
Theorem 7. The elements of the matrix $\Gamma$ are rational numbers, and the number of elements in the nth row is

$$
m(n)=\frac{1}{24}\left(2 n^{3}+15 n^{2}+37 n-30\right)-\frac{1}{4}\left[\frac{n-1}{2}\right],
$$

where $[x]$ denotes the integer part of $x$.

Proof. It is clear from the various defining equations that the coefficients of the polynomials $p_{n, j}$ are rational numbers.

A homogeneous polynomial of degree $j$ has $j+1$ terms. An $A$-polynomial of degree $2 r$ is a sum of homogeneous polynomials of even degree from 0 to $2 r$ inclusive; it consists of

$$
\sum_{l=0}^{r}(2 l+1)=(r+1)^{2}
$$

terms. Similarly, an $A$-polynomial of degree $2 r+1$ has

$$
\sum_{l=1}^{r+1} 2 l=r^{2}+3 r+2
$$

terms.

Equation (4.6a) shows that $p_{n, 0}$ consists of a single nonzero term. For $i \geq 2$, the polynomial $p_{n, i-1}$, being an $A$-polynomial of degree $i$, has $\frac{1}{4}(i+2)^{2}$ terms if $i$ is even, and $\frac{1}{4}(i+2)^{2}-\frac{1}{4}$ terms if $i$ is odd. For a given $n$, the total number of terms is

$$
\begin{aligned}
m(n) & =1+\frac{1}{4} \sum_{i=2}^{n}(i+2)^{2}-\frac{1}{4}\left[\frac{n-1}{2}\right] \\
& =\frac{1}{24}\left(2 n^{3}+15 n^{2}+37 n-30\right)-\frac{1}{4}\left[\frac{n-1}{2}\right] .
\end{aligned}
$$

A computer algebra system may be used to compute the polynomials $p_{n, j}$ from (4.6), as polynomials in $s$ and $u$. We have used Mathematica and REDUCE for this purpose. The polynomials $p_{n-1, j}$, for $j=0(1) n-1$ and $n=0(1) 15$, are listed in the Supplement section of this issue, where $p_{n-1, j}$ is denoted by $p(j)$, for each value of $n$. The results given there may be used with (4.2), (4.3) and (4.5), to construct the Faber polynomials of degree $\leq 15$.

The first ten rows of the matrix $\Gamma$ have the form

$$
\left(\begin{array}{cccccccccccc}
1 & & & & & & & & & & & \\
2 & -2 & 1 / 2 & 1 & -1 / 2 & & & & & & & \\
3 & -3 & 3 / 4 & 3 / 2 & 3 / 4 & -4 & 1 & 1 & 1 / 2 & -1 & 1 / 2 & \\
4 & -4 & 1 & 2 & 3 & -16 / 3 & -8 / 3 & 4 / 3 & 5 / 3 & 2 / 3 & 1 / 3 & \ldots \\
5 & -5 & 5 / 4 & 5 / 2 & 25 / 4 & -20 / 3 & -25 / 3 & 5 / 3 & 10 / 3 & 10 / 3 & 5 / 3 & \ldots \\
6 & -6 & 3 / 2 & 3 & 21 / 2 & -8 & -16 & 2 & 11 / 2 & 7 & 11 / 2 & \ldots \\
7 & -7 & 7 / 4 & 7 / 2 & 63 / 4 & -28 / 3 & -77 / 3 & 7 / 3 & 49 / 6 & 35 / 3 & 77 / 6 & \ldots \\
8 & -8 & 2 & 4 & 22 & -32 / 3 & -112 / 3 & 8 / 3 & 34 / 3 & 52 / 3 & 74 / 3 & \ldots \\
9 & -9 & 9 / 4 & 9 / 2 & 117 / 4 & -12 & -51 & 3 & 15 & 24 & 42 & \ldots \\
10 & -10 & 5 / 2 & 5 & 75 / 2 & -40 / 3 & -200 / 3 & 10 / 3 & 115 / 6 & 95 / 3 & 395 / 6 & \ldots
\end{array}\right)
$$


and other entries may be read from the Supplement. As Gatermann et al. [18] found for circular sectors, all entries in a column of $\Gamma$ are expressible in terms of a polynomial in the row index. For example, we find

$$
\begin{array}{ll}
\gamma_{n 1}=n, & \gamma_{n 7}=\frac{10}{3} n-n^{2}, \\
\gamma_{n 2}=-n, & \gamma_{n 8}=\frac{1}{3} n, \\
\gamma_{n 3}=\frac{1}{4} n, & \gamma_{n 9}=-\frac{7}{12} n+\frac{1}{4} n^{2}, \\
\gamma_{n 4}=\frac{1}{2} n, & \gamma_{n 10}=-\frac{11}{6} n+\frac{1}{2} n^{2}, \\
\gamma_{n 5}=\frac{1}{2} n^{2}-\frac{5}{4} n, & \gamma_{n 11}=\frac{29}{12} n-\frac{5}{4} n^{2}+\frac{1}{6} n^{3} . \\
\gamma_{n 6}=-\frac{4}{3} n, &
\end{array}
$$

\section{Numerical eVAluation of $a, b$, AND $\rho$}

To compute the Faber polynomials for a particular sector of an annulus, we need to evaluate the Schwarz-Christoffel parameters $(a$ and $b)$ and the transfinite diameter $\rho$. Given $R$ and $\theta$ defining a particular sector, $a$ and $b$ are found by solving the pair of nonlinear equations (2.7) and (2.8). We used a modified Newton iteration in which the partial derivatives in the Jacobian were approximated by central difference formulae of the form

$$
\frac{\partial f(a, b)}{\partial a} \approx \frac{f(a+D, b)-f(a-D, b)}{2 D} .
$$

The convergence of the Newton iteration depends on having sufficiently good initial estimates of $a$ and $b$. Table 1 gives suitable values for certain ranges of $R$ and $\theta$. As $\theta$ tends to 0 or $\pi$, and as $R$ tends to 0 or 1 , the convergence becomes much more sensitive to the choice of starting value, but the corresponding regions, which are close to known limits, are less likely to be of practical interest than those covered by Table 1 .

At each step of the Newton iteration it is necessary to evaluate the integrals in (2.7) and (2.8) numerically to an accuracy consistent with that required in the Newton iteration. Despite the square root singularities at the endpoints of the integration intervals, the NAG routine D01AHF, which is based on the GaussKronrod-Patterson family of formulae, works satisfactorily. Figures 3 and 4 show $a$ and $b$, respectively, as functions of $R$ and $\theta$, for $0.001 \leq R \leq 0.999$ and $0.3 \leq \theta \leq 3.1414$.

The integral in equation (2.17), which defines the transfinite diameter, must also be evaluated numerically to whatever accuracy we require. Again. the NAG routine D01AHF is appropriate. 
TABLE 1. Starting values for modified Newton iteration

\begin{tabular}{|c|c|c|c|c|c|}
\hline$\theta \quad R$ & $0.999-0.1$ & $0.1-0.02$ & $0.02-0.01$ & $0.01-0.005$ & $0.005-0.001$ \\
\hline $3.1414-3.0$ & $\begin{array}{c}a=0.489 \\
b=0.513 \\
D=1.0 \times 10^{-6}\end{array}$ & $\begin{array}{c}a=0.3760 \\
b=0.3761 \\
D=1.0 \times 10^{-6}\end{array}$ & $\begin{array}{c}a=0.3107 \\
b=0.3108 \\
D=1.0 \times 10^{-7}\end{array}$ & $\begin{array}{c}a=0.26591 \\
b=0.26592 \\
D=1.0 \times 10^{-8}\end{array}$ & $\begin{array}{c}a=0.17774 \\
b=0.17775 \\
1.0 \times 10^{-9}\end{array}$ \\
\hline $3.0-1.9$ & $\begin{array}{l}a=0.49 \\
b=0.52 \\
D=0.001\end{array}$ & $\begin{aligned} a & =0.12 \\
b & =0.24 \\
D & =0.001\end{aligned}$ & $\begin{aligned} a & =0.09 \\
b & =0.12 \\
D & =0.001\end{aligned}$ & $\begin{aligned} a & =0.07 \\
b & =0.12 \\
D & =0.001\end{aligned}$ & $\begin{aligned} a & =0.07 \\
b & =0.10 \\
D & =0.001\end{aligned}$ \\
\hline $1.9-1.57$ & $\begin{aligned} a & =0.12 \\
b & =0.24 \\
D & =0.001\end{aligned}$ & $\begin{aligned} a & =0.12 \\
b & =0.24 \\
D & =0.001\end{aligned}$ & $\begin{aligned} a & =0.06 \\
b & =0.10 \\
D & =0.001\end{aligned}$ & $\begin{array}{c}a=0.06 \\
b=0.10 \\
D=0.0001\end{array}$ & $\begin{array}{c}a=0.01 \\
b=0.02 \\
D=0.0001\end{array}$ \\
\hline $1.57-1.00$ & $\begin{aligned} a & =0.05 \\
b & =0.16 \\
D & =0.001\end{aligned}$ & $\begin{aligned} a & =0.03 \\
b & =0.08 \\
D & =0.001\end{aligned}$ & $\begin{aligned} a & =0.01 \\
b & =0.03 \\
D & =0.001\end{aligned}$ & $\begin{array}{c}a=0.01 \\
b=0.03 \\
D=0.0001 \\
\end{array}$ & $\begin{array}{c}a=0.0005 \\
b=0.001 \\
D=0.0001\end{array}$ \\
\hline $1.00-0.75$ & $\begin{array}{c}a=0.05 \\
b=0.07 \\
D=1.0 \times 10^{-5}\end{array}$ & $\begin{aligned} a & =0.03 \\
b & =0.08 \\
D & =0.001\end{aligned}$ & $\begin{array}{l}a=6.0 \times 10^{-3} \\
b=6.0 \times 10^{-4} \\
D=1.0 \times 10^{-5}\end{array}$ & $\begin{array}{l}a=4.0 \times 10^{-3} \\
b=3.0 \times 10^{-4} \\
D=1.0 \times 10^{-5}\end{array}$ & $\begin{array}{l}a=4.0 \times 10^{-6} \\
b=3.0 \times 10^{-3} \\
D=1.0 \times 10^{-6}\end{array}$ \\
\hline $0.75-0.50$ & $\begin{array}{c}a=0.01 \\
b=0.06 \\
D=1.0 \times 10^{-5}\end{array}$ & $\begin{aligned} a & =0.03 \\
b & =0.08 \\
D & =0.001\end{aligned}$ & $\begin{array}{l}a=6.0 \times 10^{-3} \\
b=6.0 \times 10^{-4} \\
D=1.0 \times 10^{-3}\end{array}$ & $\begin{array}{l}a=4.0 \times 10^{-3} \\
b=3.0 \times 10^{-4} \\
D=1.0 \times 10^{-5}\end{array}$ & $\begin{array}{l}a=4.0 \times 10^{-6} \\
b=3.0 \times 10^{-5} \\
D=1.0 \times 10^{-6}\end{array}$ \\
\hline $0.50-0.30$ & $\begin{array}{l}a=1.1 \times 10^{-1} \\
b=1.6 \times 10^{-3} \\
D=1.0 \times 10^{-5}\end{array}$ & $\begin{array}{l}a=5.0 \times 10^{-6} \\
b=5.0 \times 10^{-5} \\
D=1.0 \times 10^{-6}\end{array}$ & $\begin{array}{l}a=1.0 \times 10^{-6} \\
b=1.0 \times 10^{-5} \\
D=1.0 \times 10^{-7}\end{array}$ & $\begin{array}{l}a=1.0 \times 10^{-7} \\
b=1.0 \times 10^{-6} \\
D=1.0 \times 10^{-8}\end{array}$ & $\begin{aligned} a & =1.0 \times 10^{-9} \\
b & =8.0 \times 10^{-9} \\
D & =1.0 \times 10^{-10}\end{aligned}$ \\
\hline
\end{tabular}

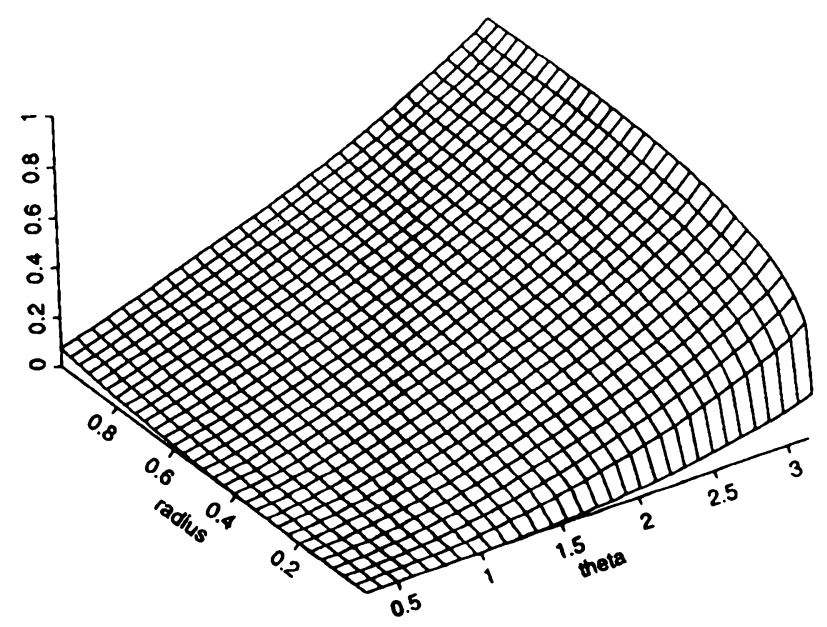

FiguRE 3. A graph of $a(R, \theta)$ 


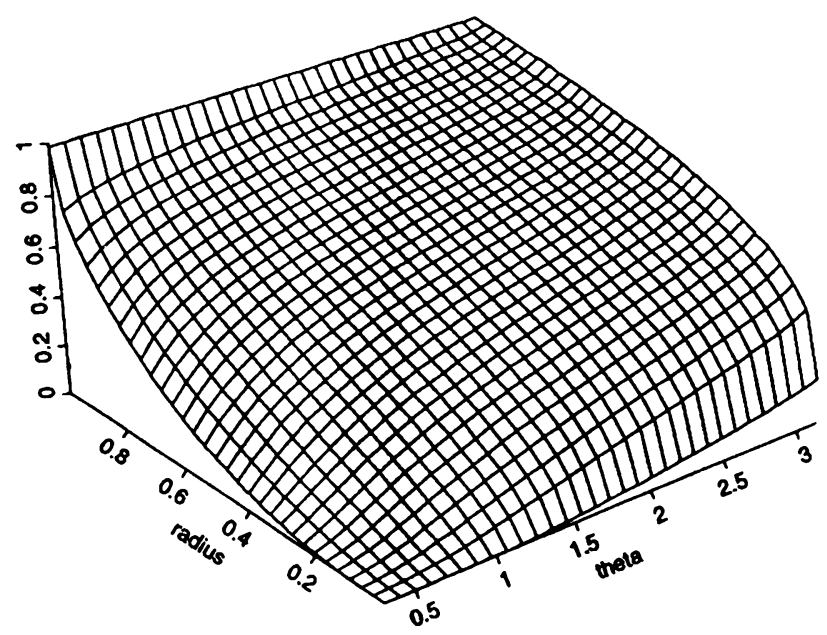

FigURE 4. A graph of $b(R, \theta)$

\section{NORMS OF FABER POLYNOMIALS ON ANNULAR SECTORS}

Any assessment of the accuracy of an approximation based on Faber polynomials requires some knowledge of a relevant norm. Both the area and line versions of the 2-norm may be computed explicitly by a slight modification of the work of Gatermann et al. [18] for circular sectors. An upper bound is available for $\left\|F_{n}\right\|_{\infty}$.

6.1. The area 2-norm. The square of the relevant norm of the scaled Faber polynomial $\widetilde{F}_{n}$ is

$$
\left\|\widetilde{F}_{n}\right\|_{2}^{2}=\int_{Q_{\gamma, R, 1}}\left|\Phi_{n}\left(\frac{z}{\rho}\right)\right|^{2} d x d y=\rho^{2} \int_{Q_{\gamma, R, \rho}}\left|\Phi_{n}(u)\right|^{2} d v d w .
$$

Here, $z=x+i y, u=v+i w, z=\rho u$ and $Q_{\gamma, R, \rho}$ is an annular sector of half-angle $\gamma$, outer radius $1 / \rho$, and inner radius $R / \rho$. Using the approach of [18], we obtain

$$
\begin{aligned}
\left\|\widetilde{F}_{n}\right\|_{2}^{2}= & I_{n, n}+2 \sum_{j=0}^{n-1} p_{n-1, j} I_{n-1-j, n}+\sum_{j=0}^{n-1} p_{n-1, j}^{2} I_{n-1-j, n-1-j} \\
& +2 \sum_{j>k}^{n-1} p_{n-1, j} p_{n-1, k} I_{n-1-j, n-1-k}
\end{aligned}
$$

where

$$
I_{i, j}= \begin{cases}2 \rho^{-i-j} \frac{\sin (i-j) \alpha}{(i-j)}\left[\frac{1-R^{i+j+2}}{i+j+2}\right] & \text { if } i \neq j \\ \frac{\rho^{-2 i}\left(1-R^{2 i+2}\right) \alpha}{i+1} & \text { if } i=j\end{cases}
$$


As $R \rightarrow 0$ these expressions reduce to those of $\S 3.1$ of [18], when account is taken of the difference in notation mentioned after equation (4.4) above.

6.2. The line 2-norm. In defining the line 2-norm, $\| \widetilde{F}_{n} \hat{\|}$, the surface integral of (6.1) is replaced by a line integral around the boundary of the annular sector. The square of that norm is obtained by replacing each $I_{i, j}$ in (6.2) by

$$
\hat{I}_{i, j}= \begin{cases}2 \rho^{-i-j}\left[\left(1-R^{i+j+1}\right)\left(\frac{\cos (i-j) \alpha}{i+j+1}\right)+\frac{\sin (i-j) \alpha}{(i-j)}\left(R^{i+j+1}+1\right)\right] & \text { if } i \neq j, \\ 2 \rho^{-2 i}\left[\left(\frac{1-R^{2 i+1}}{2 i+1}\right)+\alpha\left(R^{2 i+1}+1\right)\right] & \text { if } i=j .\end{cases}
$$

6.3. The maximum norm. Upper and lower bounds may be obtained for the norm

$$
\left\|\widetilde{F}_{n}\right\|_{\infty}=\max _{z \in Q}\left|\widetilde{F}_{n}(z)\right|
$$

of a scaled Faber polynomial; by the maximum principle, that maximum value occurs on the boundary of the domain $Q$. Let

$$
T_{n}(z)=z^{n}+a_{n-1} z^{n-1}+\cdots+a_{0}
$$

be the Chebyshev polynomial of degree $n$ for the annular sector $Q$, the monic polynomial of smallest maximum modulus on $Q$. It is known (Walsh [30, p. 320]) that $\left\|T_{n}\right\|_{\infty} \geq \rho^{n}$ and therefore, since no monic polynomial of degree $n$ can have smaller norm, $\left\|\widetilde{F}_{n}\right\|_{\infty} \geq 1$. An upper bound, independent of the degree $n$, comes from the inequality

$$
\left\|\widetilde{F}_{n}\right\|_{\infty} \leq \frac{V}{\pi}
$$

(see Ellacott [9]), where $V$ is the total rotation of the boundary of the domain $Q$. For an annular sector of interior angle $2 \gamma$,

$$
V=\left\{\begin{array}{l}
2 \pi+4 \gamma \text { for } 0 \leq \gamma \leq \frac{\pi}{2} \\
6 \pi-4 \gamma \text { for } \frac{\pi}{2} \leq \gamma \leq \pi
\end{array}\right.
$$

Combining these bounds, we have

$$
1 \leq\left\|\widetilde{F}_{n}\right\|_{\infty} \leq \frac{V}{\pi} .
$$

Starke and Varga [29], who used a different normalization for the Faber polynomials, provided bounds in terms of the norm of the corresponding Chebyshev polynomials. Their Theorem 3.4, for nonconvex regions, is applicable to annular sectors, and $\phi(0)$, which appears in their bounds, is given in closed form by equation (7.3) below.

\section{THE FABER SERIES FOR $z^{-1}$ ON AN ANNULAR SECTOR}

The Faber series for a function $f$, analytic in the annular sector $Q$, is an expression of the form

$$
\sum_{j=0}^{\infty} a_{j} F_{j}(z)
$$


See Curtiss [6], Markushevich [24, v. 3, p. 109], or Gaier [17, p. 44]. The coefficients are

$$
a_{j}=\frac{1}{2 \pi i \rho^{j}} \int_{|w|=R_{1}} \frac{f(\psi(w))}{w^{j+1}} d w,
$$

where $R_{1}>1$ is sufficiently small that $f$ can be extended analytically to the closed region bounded by the image under $\psi$ of the unit circle $|w|=R_{1}$. In particular, when $f(z)=z^{-1}$, the Faber series is

$$
\frac{1}{z}=-\frac{1}{w^{*} \psi^{\prime}\left(w^{*}\right)}\left[1+\sum_{n=1}^{\infty} \frac{\widetilde{F}_{n}(z)}{\left(w^{*}\right)^{n}}\right],
$$

where $\widetilde{F}_{n}(z)$ is the scaled Faber polynomial introduced in (4.2), and $w^{*}$ is the root of magnitude greater than 1 of the equation $\psi(w)=0$; in other words, $w^{*}$ is the point which $\psi$ maps to the origin in the $z$-plane. Equation (7.2) may be established either by applying Cauchy's residue theorem to (7.1) or, as in Chui et al. [1], by using a generating function for Faber polynomials and the uniqueness of the Faber series. It is clear from (2.13) that $z=0$ implies $\zeta=0$, and therefore

$$
w^{*}=\frac{1+a^{2}}{1-a^{2}} .
$$

From (2.13c) and (3.2) we obtain

$$
w \psi^{\prime}(w)=\frac{R\left(1-a^{4}\right)}{4 w a^{4}} \exp \left[\int_{a^{2}}^{-\zeta} \frac{C d x}{A(x)[A(x)+B(x)]}\right] M(w, t) M(w, \tau) .
$$

Setting $\zeta=0$ in this expression, and noting that

$$
M\left(w^{*}, t\right) M\left(w^{*}, \tau\right)=\frac{4 a^{2}}{\left(1-a^{2}\right)^{2}}
$$

and

$$
\rho=\frac{\left(1-a^{4}\right)}{4} \exp \left[\int_{0}^{a^{2}} \frac{C d x}{A(x)[A(x)+B(x)]}\right]
$$

we obtain

$$
w^{*} \psi^{\prime}\left(w^{*}\right)=\frac{R\left(1-a^{4}\right)}{4 a^{2} \rho} .
$$

Then, from equation (7.2), the Faber series for $z^{-1}$ is

$$
\frac{1}{z}=-\frac{4 \rho a^{2}}{R\left(1-a^{4}\right)}\left[1+\sum_{n=1}^{\infty}\left(\frac{1-a^{2}}{1+a^{2}}\right)^{n} \widetilde{F}_{n}(z)\right] .
$$

As a check on (7.6), it can be shown that it correctly gives a known Chebyshev expansion when $b=a$ (case (i) of $\S 2.4$ ). The Faber polynomial of degree $n \geq 1$ for the interval $[-1,1]$ is $2^{1-n} T_{n}(x)$, where $T_{n}$ is the Chebyshev polynomial of degree $n$, and the corresponding polynomial for $z \in[-1,-R]$ is

$$
F_{n}(z)=2\left(\frac{1-R}{4}\right)^{n} T_{n}\left(\frac{2 z+1+R}{1-R}\right) .
$$


With the help of results from $\S 2.4$ for this particular case, the expansion (7.6) becomes

$$
\frac{1}{z}=-\frac{1}{\sqrt{R}}\left\{1+2 \sum_{n=1}^{\infty}\left(\frac{1-\sqrt{R}}{1+\sqrt{R}}\right)^{n} T_{n}\left(\frac{2 z+1+R}{1-R}\right)\right\} .
$$

The Chebyshev expansion

$$
\frac{1}{x-\delta}=-\frac{1}{\sqrt{\delta^{2}-1}}\left\{1+2 \sum_{n=1}^{\infty}\left(\delta-\sqrt{\delta^{2}-1}\right)^{n} T_{n}(x)\right\}
$$

for $\delta>1$ and $x \in[-1,1]$, may be established by a technique used, for example, by Fox and Parker [16, p. 85]. By transforming to the interval $[-1,-R]$ and letting $\delta=(1+R)(1-R)^{-1}$, we again obtain (7.7).

The maximum norm of the error in approximating $z^{-1}$ on the domain $Q$ by a truncated Faber series

$$
q_{n}(z)=-\frac{1}{w^{*} \psi^{\prime}\left(w^{*}\right)}\left(1+\sum_{k=1}^{n}\left(w^{*}\right)^{-k} \widetilde{F}_{k}(z)\right)
$$

is easily bounded. From (6.3) we obtain

$$
\left\|\frac{1}{z}-q_{n}(z)\right\|_{\infty} \leq \frac{V\left|w^{*}\right|^{-n}}{\pi\left|w^{*} \psi^{\prime}\left(w^{*}\right)\right|\left(\left|w^{*}\right|-1\right)}
$$

(see also [1]), and from (7.3)

$$
\left\|\frac{1}{z}-q_{n}(z)\right\|_{\infty} \leq \frac{2 V \rho}{\pi R\left(1+a^{2}\right)}\left(\frac{1-a^{2}}{1+a^{2}}\right)^{n} .
$$

\section{ACKNOWLEDGMENTS}

We are very grateful to Dr. R. A. Smith for his invaluable help in constructing the conformal map, and to Professor G. Opfer for a discussion which prompted the research reported here. N. J. Myers is supported by a research studentship awarded by the Science and Engineering Research Council.

\section{BIBLIOGRAPHY}

1. C.K. Chui, J. Stöckler and J.D. Ward, A Faber series approach to cardinal interpolation, Math. Comp. 58 (1992), 255-273.

2. J.P. Coleman, Complex polynomial approximation by the Lanczos $\tau$-method: Dawson's integral, J. Comput. Appl. Math. 20 (1987), 137-151.

3. 193-211.

4. J.P. Coleman and R.A. Smith, The Faber polynomials for circular sectors, Math. Comp. 49 (1987), 231-241.

5. S1-S4.

6. J.H. Curtiss, Faber polynomials and Faber series, Amer. Math. Monthly 78 (1971), 577-596.

7. M. Eiermann, On semiiterative methods generated by Faber polynomials, Numer. Math. 56 (1989), 139-156. 
8. M. Eiermann, W. Niethammer, and R.S. Varga, A study of semiiterative methods for nonsymmetric systems of linear equations, Numer. Math. 47 (1985), 505-533.

9. S.W. Ellacott, Computation of Faber series with application to numerical polynomial approximation in the complex plane, Math. Comp. 40 (1983), 575-587.

10. Chui, L.L. Schumaker, and J.D. Ward, eds.), Academic Press, New York, 1983, pp. 457464.

11. Numer. Anal. 20 (1983), 989-1000.

12. S.W. Ellacott and M.H. Gutknecht, The polynomial Carathéodory-Fejér approximation method for Jordan regions, IMA J. Numer. Anal. 3 (1983), 207-220.

13. S.W. Ellacott and E.B. Saff, Computing with the Faber transform, Rational Approximation and Interpolation (P.R. Graves-Morris, E. Saff, and R.S. Varga, eds.), Lecture Notes in Math., vol. 1105, Springer-Verlag, Berlin, 1984, pp. 412-418.

14. __ On Clenshaw's method and a generalisation to Faber series, Numer. Math. 52 (1988), 499-509.

15. G.H. Elliott, The construction of Chebyshev approximations in the complex plane, Ph.D. Thesis, University of London, 1978.

16. L. Fox and I.B. Parker, Chebyshev polynomials in numerical analysis, Oxford Univ. Press, London, 1968.

17. D. Gaier, Lectures on complex approximation, Birkhäuser, Boston, 1987.

18. K. Gatermann, C. Hoffmann, and G. Opfer, Explicit Faber polynomials on circular sectors, Math. Comp. 58 (1992), 241-253.

19. , Supplement to explicit Faber polynomials on circular sectors, Math. Comp. 58 (1992), S1-S6.

20. U. Grothkopf and G. Opfer, Complex Chebyshev polynomials on circular sectors with degree six or less, Math. Comp. 39 (1982), 599-615.

21. P. Henrici, Applied and computational complex analysis, Vol. 1, Wiley, New York, 1974.

22. __ Applied and computational complex analysis, Vol. 3, Wiley, New York, 1986.

23. T. Kövari and C. Pommerenke, On Faber polynomials and Faber expansions, Math. Z. 99 (1967), 193-206.

24. A.I. Markushevich, Theory of functions of a complex variable, Chelsea, New York, 1977.

25. N. Papamichael, M.J. Soares, and N.S. Stylianopoulos, A numerical method for the computation of Faber polynomials for starlike domains, IMA J. Numer. Anal. 13 (1993), 181-193.

26. C. Pommerenke, Über die Faberschen Polynome schlichter Funktionen, Math. Z. 85 (1964), 197-208.

27. A.P. Prudnikov, Yu.A. Brychkov, and O.I. Marichev, Integrals and series, Vol. I: Elementary functions, Gordon and Breach, New York, 1988.

28. V.I. Smirnov and N.A. Lebedev, Functions of a complex variable, constructive theory, Iliffe, London, 1968.

29. G. Starke and R.S. Varga, A hybrid Arnoldi-Faber iterative method for nonsymmetric systems of linear equations, Numer. Math. 64 (1993), .213-240.

30. J.L. Walsh, Interpolation and approximation by rational functions in the complex domain, 5th ed., Amer. Math. Soc. Colloq. Publ., vol. 20, Amer. Math. Soc., Providence, RI, 1969.

Department of Mathematical Sciences, University of Durham, South Road, Durham, DH1 3LE, ENGLAND

E-mail address: john.coleman@durham.ac.uk

Department of Mathematical Sciences, University of Durham, South Road, Durham, DH1 3LE, ENGLAND

E-mail address: n.j.myers@durham.ac.uk 


\title{
Supplement to
}

\section{THE FABER POLYNOMIALS FOR ANNULAR SECTORS}

\author{
JOHN P. COLEMAN AND NICK J. MYERS
}

\section{Appendix}

Table 2. Faber polynomials up to degree 15 Polynomials $\phi_{n-1}=\sum_{j=0}^{n-1} p_{j} z^{n-j-1}$ for $1 \leq n \leq 15$.

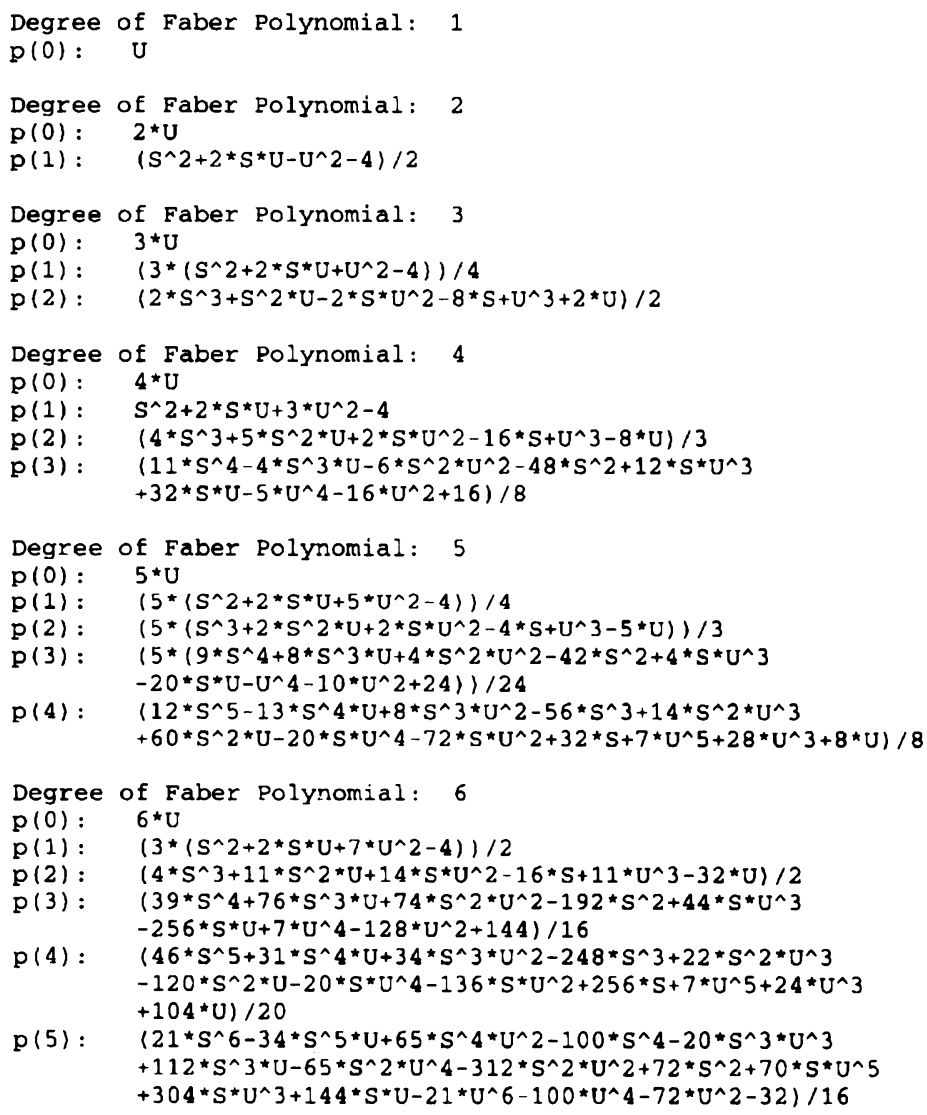



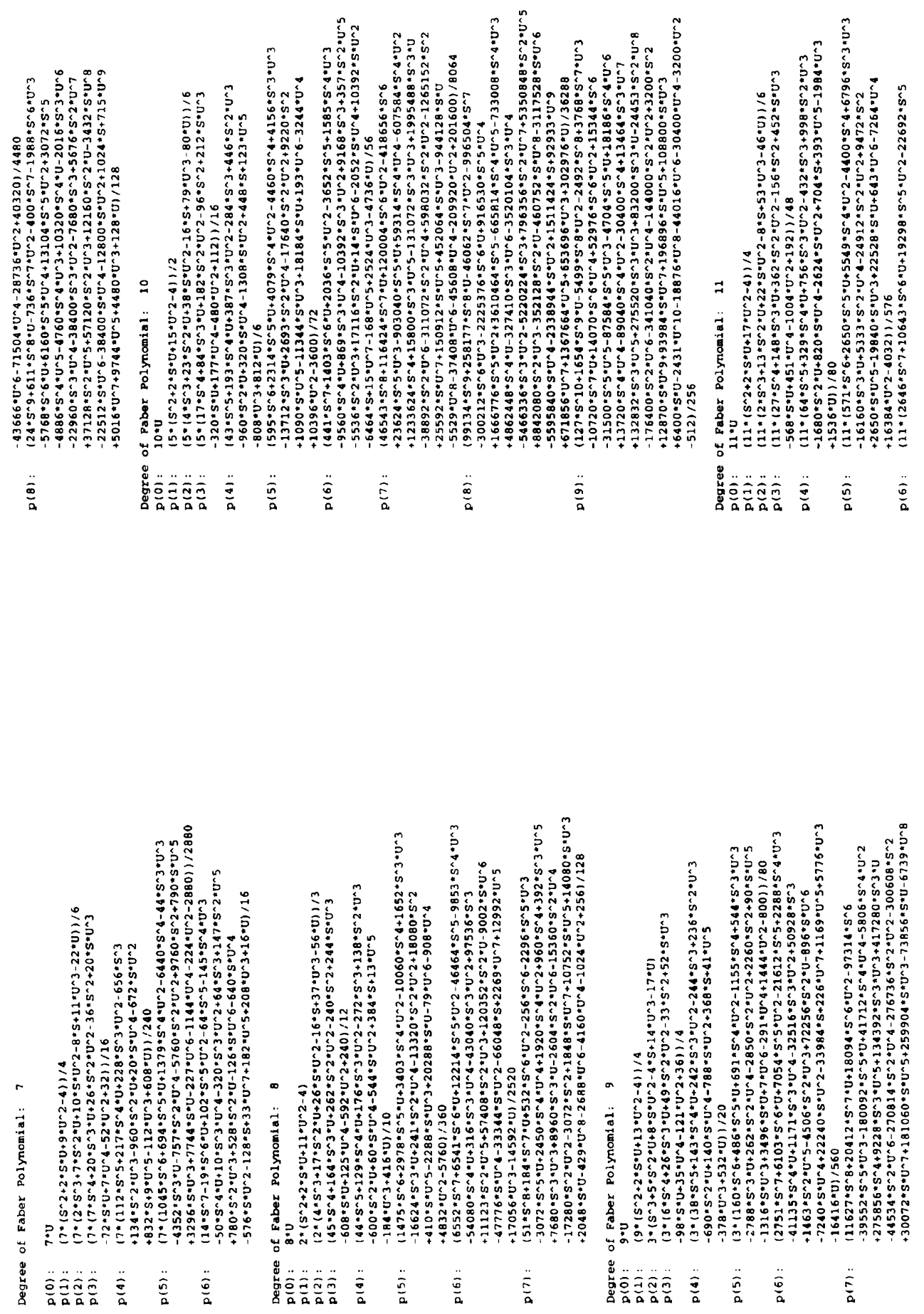

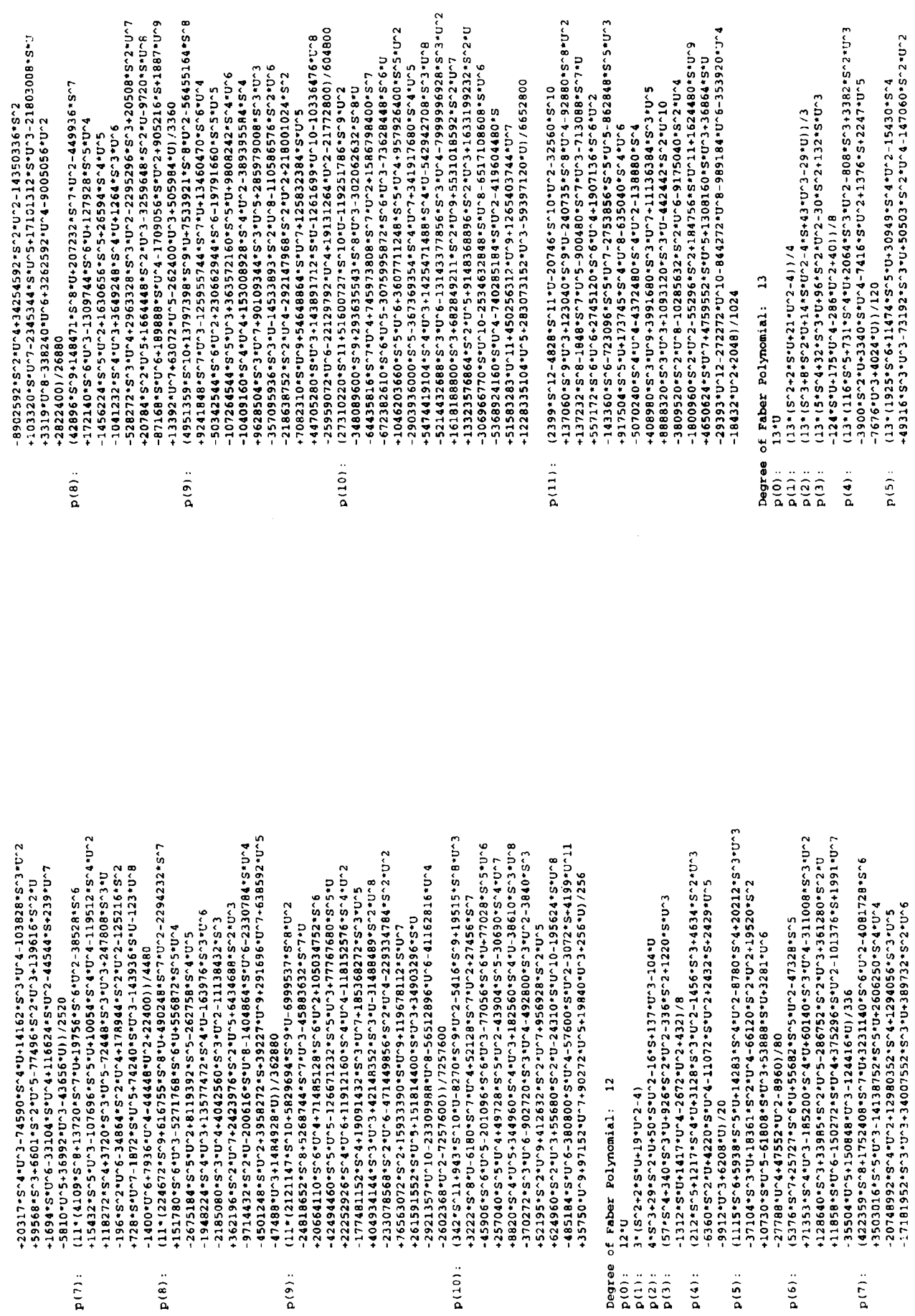

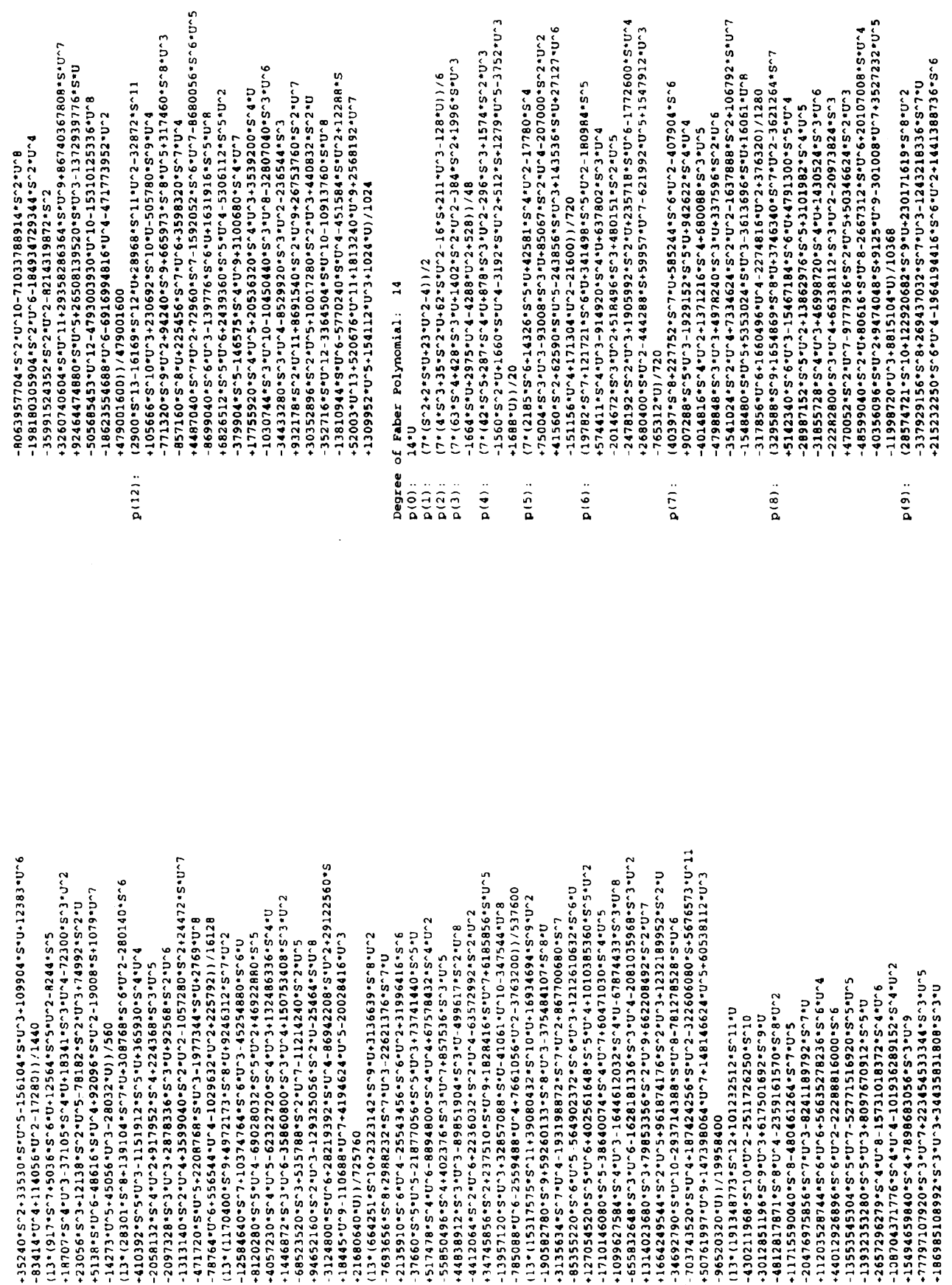

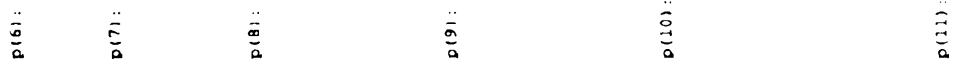



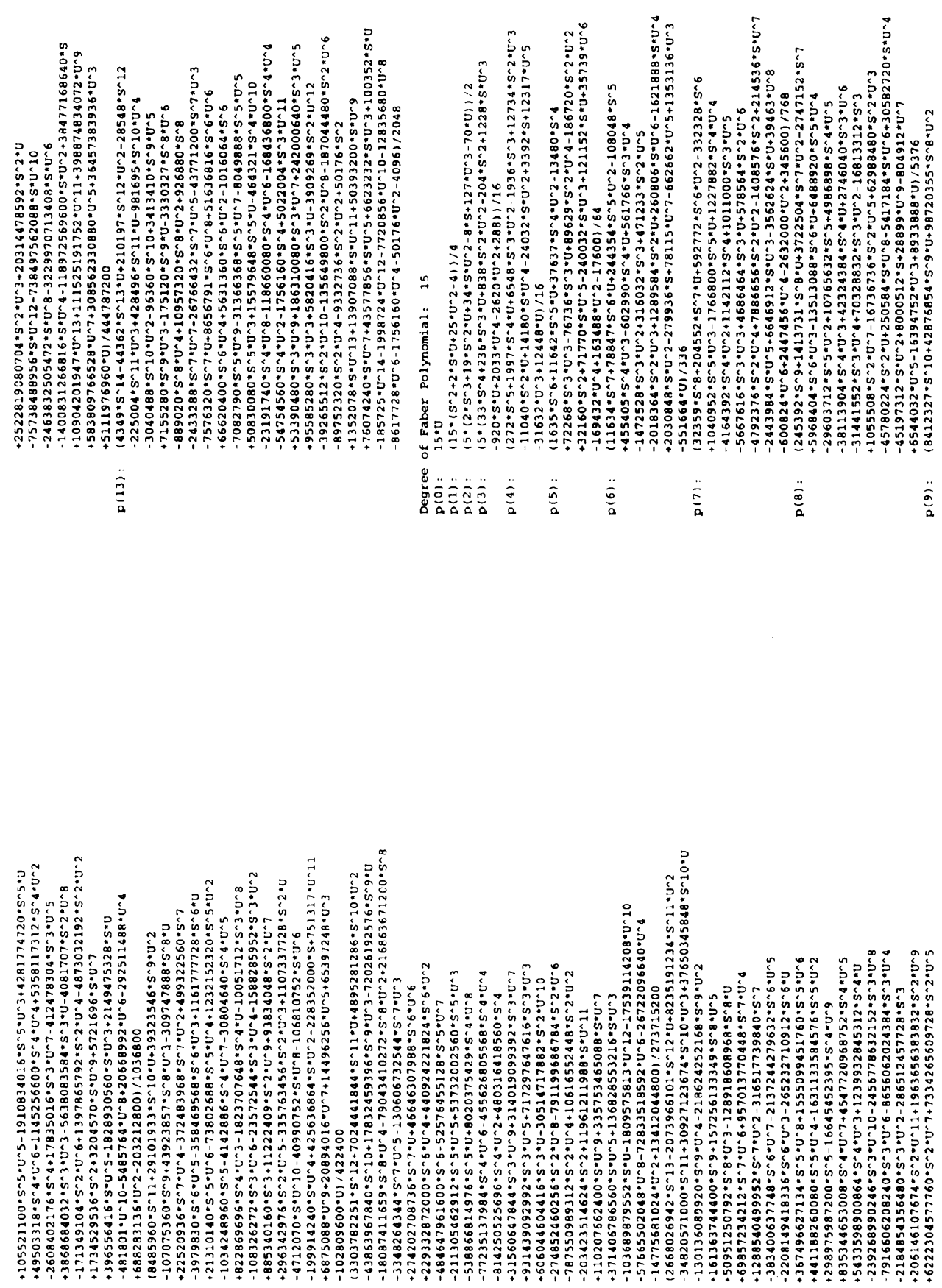

苛 


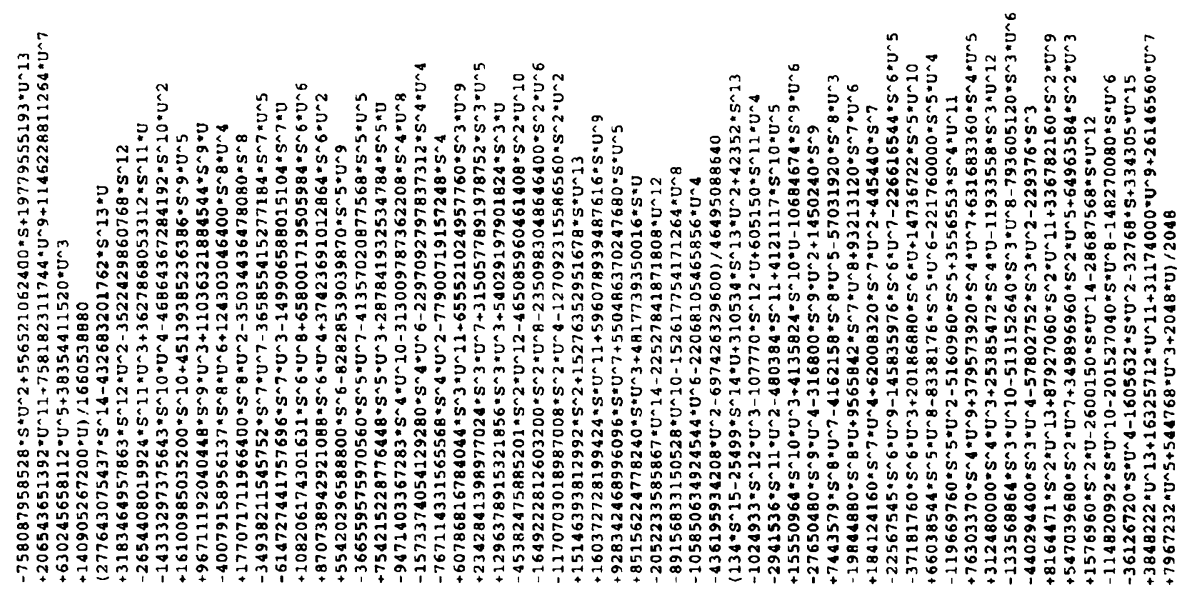

商

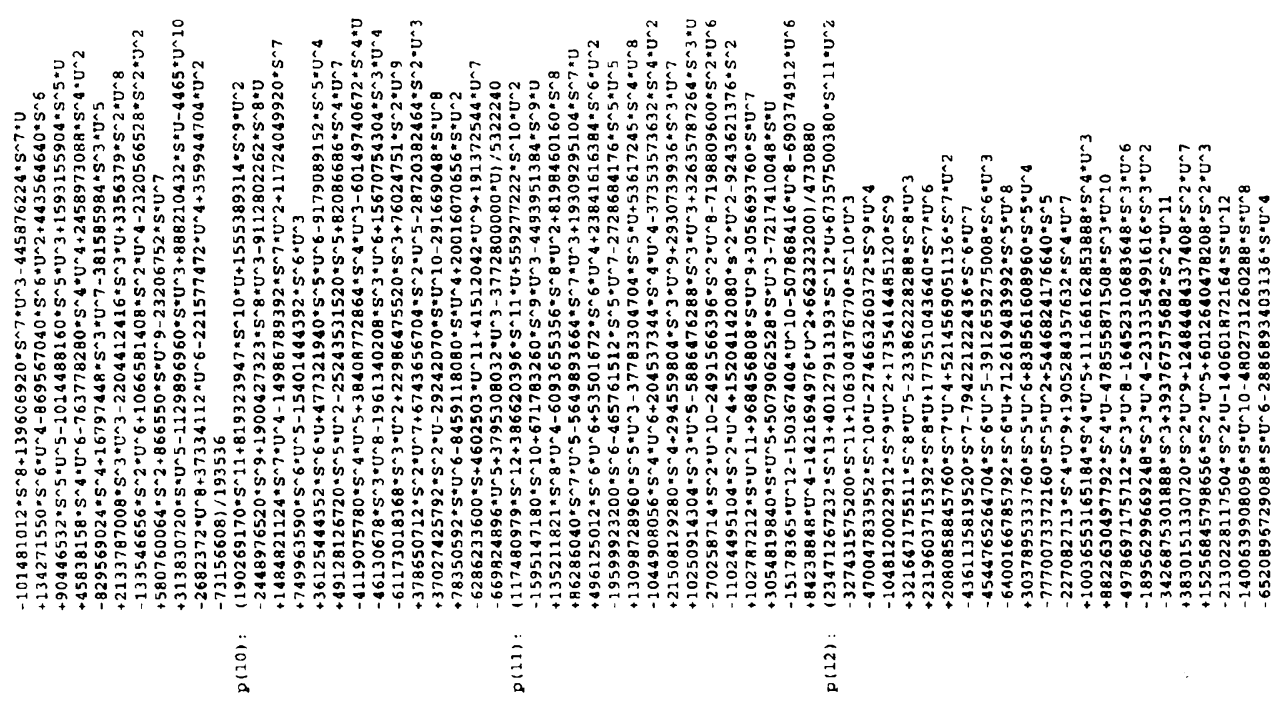

